\title{
The lin-14 locus of Caenorhabditis elegans controls the time of expression of specific postembryonic developmental events
}

\author{
Victor Ambros ${ }^{1}$ and H. Robert Horvitz \\ Department of Biology, Massachusetts Institute of Technology, Cambridge, Massachusetts 02139 USA
}

\begin{abstract}
The lin-14 locus of Caenorhabditis elegans plays an important role in specifying the normal timing and sequence of developmental events in the lateral hypodermal cell lineages. The results of gene dosage, complementation, and temperature-shift experiments indicate that the fates expressed by cells at successive stages of these cell lineages are specified by the level of lin-14 activity and that lin-14 acts at multiple times during development to control stage-specific choices of cell fate. Our observations suggest that during normal development a reduction in the level of lin-14 gene function causes the sequential expression of stage-specific cell fates.
\end{abstract}

[Key Words: lin-14; C. elegans; temperature-shifts; complex locus; developmental timing]

Received January 5, 1987; revised version received and accepted March 20, 1987.

Mutations that alter temporal patterns of cell division and differentiation have been identified in the nematode Caenorhabditis elegans (Ambros and Horvitz 1984). Like homeotic mutations of insects (Ouweneel 1976; Morata and Lawrence 1977) and nematodes (Greenwald et al. 1983; Sternberg and Horvitz 1984), these "heter-

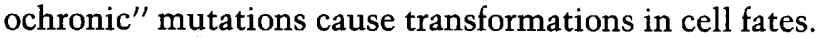
However, in contrast to homeotic mutations that cause particular cells to adopt the fates of cells at other positions, heterochronic mutations cause cells to adopt fates normally expressed by cells at earlier or later stages of development. Heterochronic mutations have been identified in four C. elegans genes-lin-4, lin-14, lin-28, and lin-29 (Chalfie et al. 1981; Ambros and Horvitz 1984). The phenotypic consequences of these mutations suggest that heterochronic genes may play important roles in normal development by controlling the times during development that specific cell fates are expressed.

The heterochronic gene $\mathrm{lin}-14$ is of particular interest because two distinct classes of lin-14 mutations have been identified that result in discrete and opposite transformations in cell fates (Ambros and Horvitz 1984). Recessive lin-14 mutations cause precocious development, i.e., certain cells adopt fates normally specific for cells generated one larval stage later in the same lineage. In contrast, semidominant lin-14 mutations cause retarded development, i.e., cells at later stages adopt fates normally expressed by cells earlier in the same lineage.

'Present address: Department of Cellular and Developmental Biology, Harvard University, Cambridge, Massachusetts 02138 USA. lin-14 mutations alter the fates of cells in a number of cell lineages and in various tissues, including the lateral hypodermis, mesoderm, and intestine of both sexes and the posterior ectoderm of the male. The effects of lin-14 are easiest to interpret in the lateral hypodermal lineages, in which each larval stage of the wild type is characterized by a distinctive pattern of cell division and differentiation. The lateral hypodermal blast cells ("seam cells" $\mid$ divide according to stage-specific patterns, which we have named $\mathrm{S} 1, \mathrm{~S} 2, \mathrm{~S} 3$, and $\mathrm{S} 4$ to correspond to the L1, L2, L3, and L4 larval stages, respectively (Ambros and Horvitz 1984). At the end of the fourth larval stage, seam cells do not divide but instead generate cuticular structures known as lateral alae. Thus, seam cells can express five possible fates: They can give rise to $S 1, S 2$, $\mathrm{S} 3$, or $\mathrm{S} 4$ cell division patterns, or they can produce lateral alae. In the wild-type lateral hypodermal lineages, these fates are expressed in the fixed temporal order S1, S2, S3, S4, alae. In lin-14 mutants, these fates are expressed aberrantly, e.g., S2, S3, S4, alae or S1, S1, S1, S1, suggesting that lin-14 activity controls the timing and sequence of fates expressed in the lateral hypodermal cell lineages (Ambros and Horvitz 1984).

To define more clearly the role of lin-14 in wild-type development, we needed to determine how mutations perturb the normal activity of the gene and how altering gene activity at specific times in development affects the fates of specific cells. In this manuscript, we describe changes in the wild-type temporal pattern of seam cell fates caused by different lin-14 alleles and heteroallelic combinations. In particular, we determine the effects of 
a. WILD TYPE

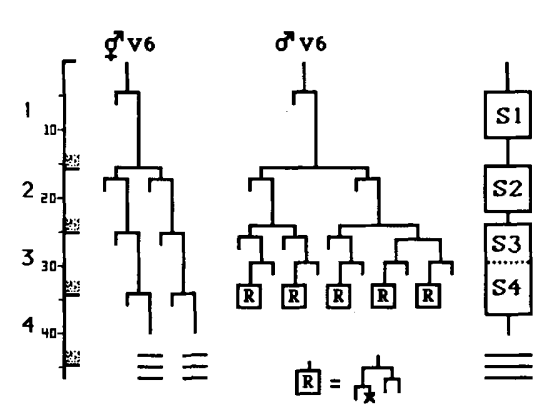

d. CLASS III $\underline{\text { in-14(n)360) }}$

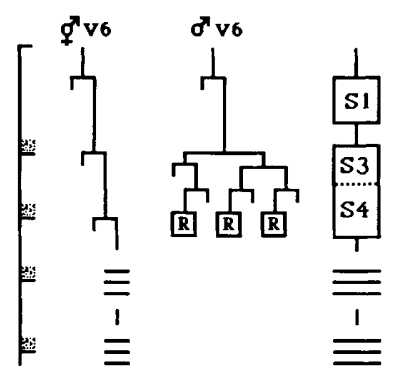

b. CLASS I lin-14(n536)

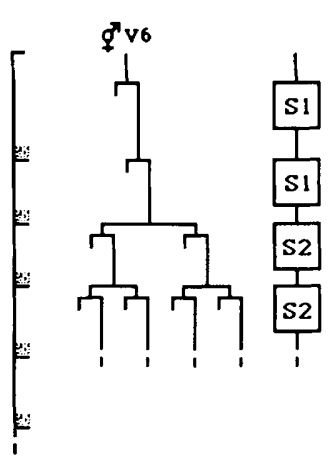

e. CLASS IV Iin-14(n536n838)

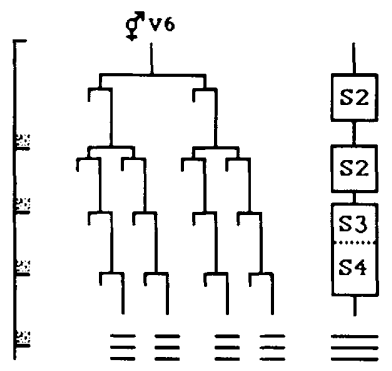

C. CLASSII lin-14(n536n540)

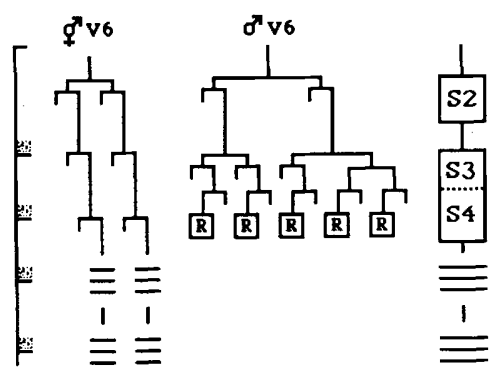

f. CLASS V lin-14(n355 0679) $15^{\circ} \mathrm{C}$

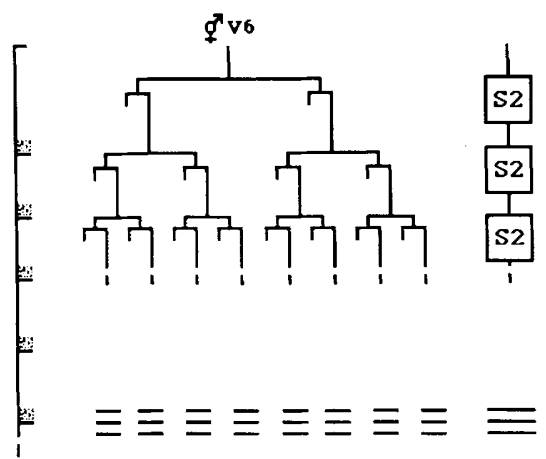

Figure 1. Heterochronic lateral hypodermal cell lineage patterns of different phenotypic classes of lin-14 mutants. Each mutant represents an example of one of the five phenotypic classes listed in Table 1. Cell lineages were followed as described by Sulston and Horvitz (1977). The vertical columns of boxes refer to the lineage patterns S1-S4 that compose each lineage in the wild type. Each lineage pattern is expressed at a particular stage in the wild type and can be expressed at other stages in heterochronic mutants (Ambros and Horvitz 1984). S1, S3, and S4 patterns appear similar in hermaphrodites, but are distinguishable in males. The time of adult lateral alae formation, which occurs at the fourth molt in the wild type, is indicated by the triple horizontal bar. Except for the $n 355 \mathrm{n} 679$ animal maintained at $15^{\circ} \mathrm{C}$ (see below), each mutant lineage is from a single animal maintained at $20^{\circ} \mathrm{C}$ in which every cell division was observed. Although the cell lineages of these mutants are somewhat variable, the patterns shown here are representative of each strain. (a) Lineages generated by postembryonic blast cell V6 of the wild-type hermaphrodite and male (from Sulston and Horvitz 1977). V6 is one of a set of similar lateral hypodermal blast cells positioned along the lateral line or "seam" of the animal. In this paper, we consider the set of normally identical lineages derived from the lateral hypodermal blast cells V1-V4 and V6 in hermaphrodites as well as from V6 in males. In the wild type, the seam cell progeny of these cells cease cell division after the L4 stage and at the L4 molt generate the lateral alae (Singh and Sulston 1978). (b) lin-14(n536), class I phenotype; semidominant, retarded mutant. S1 lineage patterns are reiterated and S2 lineage patterns are delayed and/or reiterated. Supernumerary molts occur. Cell division patterns at supernumerary stages were not followed in detail but appeared similar to the S3 and S4 patterns of the wild type. The cuticle formed at the L4 and subsequent molts appears unlike normal adult cuticle and more like larval cuticle both in its ultrastructural layering pattern and the absence of normally adult-specific lateral alae (Ambros and Horvitz 1984). In some animals of this genotype, a small proportion of seam cells form alae at the normal time (L4 molt). Alae are also formed by some seam cells at supernumerary stages. More severe retarded defects are caused by lin-14(n355). In lin-14(n355) animals, S1 lineage patterns are reiterated for as many as four stages, and consequently S2 patterns rarely occur (Ambros and Horvitz 1984). (c) lin-14(n536 n540), class II phenotype; recessive, precocious mutant. S1 lineage patterns are deleted. S2, S3, and S4 cell lineage patterns and adult alae formation are precocious by one larval stage. Adult alae formation occurs twice; at the L3 molt and again at the normal time (L4 molt). (d) lin-14(n360), class III phenotype; recessive, precocious mutant. S3 and S4 cell lineage patterns and adult alae formation are precocious by one larval stage. S2 lineage patterns are deleted. Like class II animals, these animals form adult alae at successive stages, the L3 and L4 molts. (e) lin-14(n536 n838), class IV phenotype. This strain is a partial revertant of the semidominant mutant lin-14(n536). lin-14(n536 n838) animals display precocious S2 lineage patterns and deletion of S1 lineage patterns. In the lineages shown here, subsequent development paralleled that of the wild type. Occasionally, these animals can also display precocious adult alae formation (not shown; see Tables 1 and 3). (f) lin-14(n355 n679ts) at $15^{\circ} \mathrm{C}$, class V phenotype. At $15^{\circ} \mathrm{C}$ lin-14(n355 n679) animals display retarded developmental defects (see Figs. 2, 3, and 6; Tables 1 and 4), as well as precocious S2 lineage patterns. Lineages were followed by intermittent observation at $20^{\circ} \mathrm{C}$, with animals maintained at $15^{\circ} \mathrm{C}$ between observations. Lineages were not followed in detail after the L3 stage, but in this animal, and in other animals of the same genotype, seam cells were observed to divide at the L4 molt and form adult alae at the fifth molt, as shown here. 
altering the dosage of wild-type lin-14 gene activity. We also define the periods of lin-14 activity from temperature-shift experiments utilizing temperature-sensitive alleles. Our studies strongly suggest that the level of lin-14 activity (e.g., "high" vs. "low") specifies the fates expressed by certain cells, and that during development lin-14 activity decreases, thereby causing different cell fates to be expressed at successive developmental stages. Our experiments indicate that the lin-14 locus specifies at least two distinct functions that control different stage-specific choices of cell fates within the lateral hypodermal cell lineages.

\section{Results}

\section{Five classes of lin-14 alleles}

We have isolated 19 alleles of the gene lin-14 (Ambros and Horvitz 1984). These alleles define five classes based on the developmental defects that they cause (Fig. 1; Table 1):

Class I alleles are semidominant. Animals heterozygous or homozygous for 1 in-14(n536) or lin-14(n355) display retarded expression of normally stage-specific cell lineage patterns, retarded larval cuticle formation, and supernumerary molts (Ambros and Horvitz 1984; Fig. 1b). Heterozygous animals display the same defects as homozygous animals but with lesser severity (see below, Tables 1 and 4 l.

Class II alleles are recessive and cause precocious expression of S2, S3, and S4 cell lineage patterns and precocious adult alae formation (Fig. 1c). Certain class II alleles appear to be null alleles, causing complete loss of function (see below).

Class III alleles are recessive and cause a subset of the defects caused by class II alleles, i.e., they cause precocious S3, S4, and adult alae formation, but they do not cause precocious S2 lineage patterns (Fig. 1d; Table 1).

Class IV has a single member, $n 536 n 838$. This mutant contains a semidominant class I allele, $n 536$, and a closely linked recessive mutation, n838. n536 n838 animals display precocious S2 cell lineage patterns and, in rare cases, precocious adult alae formation (Fig. 1e; Table 1).

Class V is defined by two alleles, $n 536 n 538$ and $n 355$ n679. $n 536 n 538$ animals and $n 355 n 679$ animals display complex phenotypes with both retarded and precocious defects (e.g., see Fig. 1f). Presumably, the retarded defects of these mutants are caused by the class I mutations, $n 536$ and $n 355$, respectively, and the precocious defects are caused by the closely linked mutations, $n 538$ and $n 679$, respectively. The $n 679$ mutation seems to be a temperature-sensitive suppressor of $n 355$. Specifically, the retarded defects of $n 355 n 679$ animals grown at $15^{\circ} \mathrm{C}$ are more severe than those of animals grown at $20^{\circ} \mathrm{C}$. n355 n679 animals also display a corresponding increased strength of precocious defects with increased temperature. For both class $\mathrm{V}$ alleles, certain retarded defects are expressed in heterozygotes (Table 1).

The defects displayed by the five classes of lin-14 mutants (Fig. 1; Table 1) seem to involve temporal transfor- mations in cell fates (Ambros and Horvitz 1984). For example, retarded S2 lineage patterns result from the expression of S2 patterns instead of S3 patterns by seam cells in the L3 stage (Fig. 1b,f). Conversely, precocious S2 patterns result from the expression of S2 patterns instead of S1 patterns by seam cells in the L1 stage (Fig. $1 \mathrm{c}, \mathrm{f})$. The cell fate transformations corresponding to the other precocious and retarded defects are described in the legends to Table 1 and Figure 1.

\section{Precocious defects are caused by a loss of lin-14 activity}

The genetic properties of class II lin-14 alleles are consistent with their causing a reduction or loss of gene function. First, class II lin-14 alleles are recessive and are the most common class of closely linked cis-acting suppressors of lin-14 semidominant mutations. Furthermore, several class II alleles seem to be null alleles (i.e., causing a complete loss of gene function) based on two criteria: (1) they lead to the most extreme precocious phenotype observed, and (2) they act like a deficiency of lin-14 in heteroallelic combinations.

The recessive alleles that cause the most extreme phenotype were identified by quantifying the severity of the heterochronic defects of different lin-14 mutants. This quantitation was done by scoring the transformations in cell fates caused by lin-14 mutations; these transformations are discrete and are detectable at the level of individual cells (e.g., Fig. 2). By determining the fates of large numbers of individual seam cells at specific stages of development, we have determined the frequency of the expression of specific heterochronic cell fates in strains carrying various lin-14 allelic combinations (see Scoring seam cell fates section of Materials and methods). We have used this frequency as a measure of the severity of mutant phenotypes.

Mutations of the same phenotypic class can differ in the severity of their phenotypes. Based on the frequency of two precocious defects, precocious S2 lineage patterns and precocious adult alae formation, five members of the major class of recessive alleles appear to be the most strongly affected. For example, the frequency of expression of both of these defects is $90-100 \%$ for the severe allele lin-14(n536 n540) but about $30 \%$ for the weaker allele lin-14(n179ts) at $20^{\circ} \mathrm{C}$ (Table 2). Whereas the other class II alleles cause a range of relatively milder phenotypes, these five class II alleles (n536 n540, n536 n837, $n 536 n 842, n 355 n 535, n 355 n 726)$ result in the most severe precocious phenotype and hence are the most likely among the existing alleles to cause a complete loss of lin-14 function.

Additional evidence that these severe class II alleles are lin-14 null alleles is that they have genetic properties identical to those of $n D f 19$, a deficiency that deletes the lin-14 locus (Ambros and Horvitz 1984). First, like $n D f 19$ (see Table 2), the severe alleles are completely recessive (data not shown). Second, these severe mutations and $n D f 19$ similarly enhance the penetrance of the defects of a weaker allele, $n 179$ ts at $20^{\circ} \mathrm{C}$, in trans heterozygotes (Table 2). Third, these severe lin-14 alleles and 
Table 1. Heterochronic lateral hypodermal defects of lin-14 mutants

\begin{tabular}{|c|c|c|c|c|c|c|c|c|c|}
\hline \multirow[b]{3}{*}{ Class $^{a}$} & \multirow[b]{3}{*}{ Alleles ${ }^{b}$} & & \multicolumn{7}{|c|}{ Defects $^{c}$} \\
\hline & & & \multicolumn{3}{|c|}{ retarded ${ }^{\mathrm{d}}$} & \multicolumn{4}{|c|}{ precocious } \\
\hline & & & S1 & S2 & $\mathrm{S} 3, \mathrm{~S} 4$ & $\mathrm{~S} 2$ & S3 & S4 & alae \\
\hline \multirow[t]{4}{*}{ I semidominant } & n355@ & & $* \star *$ & $\star \star \star *$ & $* * \star$ & & & & \\
\hline & n355/n355 n726 & & ** & $\star \star$ & $* \star *$ & & & & \\
\hline & $n 536 @$ & & $* \star *$ & $\star \star \star$ & $* \star \star$ & & & & \\
\hline & $n 536 /+@$ & & $\star *$ & $\star \star$ & $* \star *$ & & & & \\
\hline \multirow[t]{14}{*}{ II recessive } & n536n540@ & & & & & $\star \star \star *$ & $\star \star \star$ & $\star \star \star$ & $\star \star \star *$ \\
\hline & $n 536$ n837 & & & & & $\star \star \star$ & $\star \star \star$ & nd & 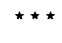 \\
\hline & $n 536 n 842$ & & & & & $\star \star \star$ & $\star \star \star$ & nd & $\star \star \star$ \\
\hline & n355 n535 & & & & & $\star \star \star *$ & $\star \star \star$ & nd & $\star \star \star$ \\
\hline & $n 355 n 726$ & & & & & $\star \star \star$ & $\star \star \star$ & nd & $\star \star \star$ \\
\hline & n536 n539 & & & & & $\star \star$ & $\star \star$ & nd & $\star \star$ \\
\hline & n355n407@ & & & & & $\star \star$ & $\star \star$ & $\star \star$ & $\star \star$ \\
\hline & n355 n531 & & & & & ** & $\star \star$ & nd & * * \\
\hline & $n 179 t s^{@}$ & $15^{\circ} \mathrm{C}$ & & & & & & & \\
\hline & n179ts & $20^{\circ} \mathrm{C}$ & & & & $\star \star$ & $\star \star$ & $\star \star$ & * * \\
\hline & n179ts & $25^{\circ} \mathrm{C}$ & & & & $\star \star \star$ & $\star \star \star$ & $\star \star \star$ & 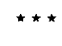 \\
\hline & n530ts & $15^{\circ} \mathrm{C}$ & & & & & & & \\
\hline & n530ts & $20^{\circ} \mathrm{C}$ & & & & $\star \star$ & $\star *$ & $\star \star$ & ** \\
\hline & n530ts & $25^{\circ} \mathrm{C}$ & & & & $\star \star \star$ & $\star \star \star$ & 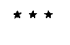 & 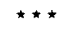 \\
\hline \multirow[t]{4}{*}{ III recessive } & n360@ & & & & & & $\star \star *$ & $\star \star \star *$ & 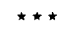 \\
\hline & $n 727^{@}$ & & & & & & $\star$ & $\star \star$ & $\star \star \star$ \\
\hline & n355 n534 & & & & & & $\star \star *$ & nd & $\star \star$ \\
\hline & n355 n840 & & & & & & * * & nd & $\star \star$ \\
\hline IV recessive & n536 n838 & & & & & $\star \star \star$ & & nd & $\star$ \\
\hline \multirow[t]{7}{*}{ V complex } & n536 n538 & & & $* *$ & ** & & & nd & $\star$ \\
\hline & n536 n538/ + & & & & * & & & & \\
\hline & n355 n679ts & $15^{\circ} \mathrm{C}$ & & $\star \star \star$ & $\star \star *$ & $\star *$ & & & \\
\hline & n355 n679ts + & $15^{\circ} \mathrm{C}$ & & $\star$ & $\star \star$ & & & & \\
\hline & n355 n679ts & $20^{\circ} \mathrm{C}$ & & & * & $\star \star *$ & & nd & $\star$ \\
\hline & n355 n679ts $/+$ & $20^{\circ} \mathrm{C}$ & & & * & & & & \\
\hline & n355 n679ts & $25^{\circ} \mathrm{C}$ & & & & $\star \star \star$ & ** & nd & $\star \star$ \\
\hline
\end{tabular}

Phenotypic classes of lin-14 mutants based on the heterochronic lateral hypodermal cell lineage defects described in Fig. 1. As described in the text and in Fig. 1, the defects displayed by these five classes of lin-14 mutants seem to involve temporal transformations in cell fates (Ambros and Horvitz 1984). Retarded S1 lineage patterns result from the expression of S1 patterns instead of S2 patterns by cells in the L2; retarded S2 patterns result from the expression of S2 patterns instead of S3 patterns by cells in the L3; retarded S3 or S4 cell lineage patterns result from the expression of S3 or S4 lineage patterns instead of adult alae formation by cells at the L4 molt (retarded defects were characterized in hermaphrodites, where S3 and S4 lineage patterns are not distinguishable); precocious S2 patterns result from the expression of S2 patterns instead of S1 patterns by cells in the L1; precocious S3, S4, and alae result from the expression of those fates instead of S2, S3, and S4 lineage patterns by cells in the L2, L3, and L3 molt, respectively.

a Mutants are classified (as class I, II, III, IV, or V) according to the defects that were observed. For each mutant described here as lacking a particular defect, that defect was not observed after examination of at least 20 animals.

b The defects caused by certain alleles of each class (@) were determined by observing every cell division of the lateral hypodermal cell lineages of blast cells V1 $\sim \mathrm{V} 6$ and $\mathrm{T}$ in at least one hermaphrodite and further confirmed by screening animals for defects in cellular anatomy known to reflect cell lineage abnormalities (see Materials and methods). The effects of other alleles of each class were characterized by similar screens for cell lineage-derived anatomical defects. Precocious S4 lineage patterns were scored by examining the V5, V6, and T lineages of males. All other determinations were made by examining hermaphrodites. ts identifies alleles that are temperature-sensitive, as described in the text and legends to Figs. 4, 5, and 6. All experiments were performed at $20^{\circ} \mathrm{C}$ unless otherwise noted. Among the lin-14 recessive alleles (classes II, III, and IV) are lin-14 single mutations and presumed lin-14 double mutations. These double mutations were derived by the reversion of mutants carrying lin-14 semidominant mutations (Ambros and Horvitz 1984). Such revertants are assumed to contain the original semidominant mutation and a closely linked, cis-acting suppressor of the semidominant mutation. Most of these double mutations are recessive lin-14 alleles. Since lin-14(n355 n679) and lin-14(n536 $\mathrm{n538}$ ) (class V) are partial revertants of lin-14 semidominant alleles, the complex phenotype of each of these alleles probably reflects an elevation of lin-14 activity caused by the $n 355$ or $n 536$ semidominant mutation and a loss of lin-14 activity caused by the $n 679$ or n538 mutations, respectively.

c The defects caused by the indicated alleles are shown by asterisks. All phenotypes are for homozygous animals except where otherwise indicated. The strength of each defect is indicated as follows: No asterisk indicates no defects were observed; $\left({ }^{\star}\right)$ an occasional animal displayed the indicated defect, and usually only a minority of its lateral hypodermal (i.e., V1-V6) lineages were affected; $\left(^{\star *}\right)$ most animals displayed the defect, but for the affected animals, not all V1-V6 lineages were abnormal; $\left.\left.\right|^{\star \star \star}\right)$ essentially all V1-V6 lineages of all animals displayed the defect; (nd) not determined, i.e., animals were not examined for the indicated defect. d All strains displaying retarded S3 or S4 lineage patterns also undergo at least one supernumerary molt with the exception of $n 355$ $n 679$ and $n 355 n 679 /+$ at $20^{\circ} \mathrm{C}$. 


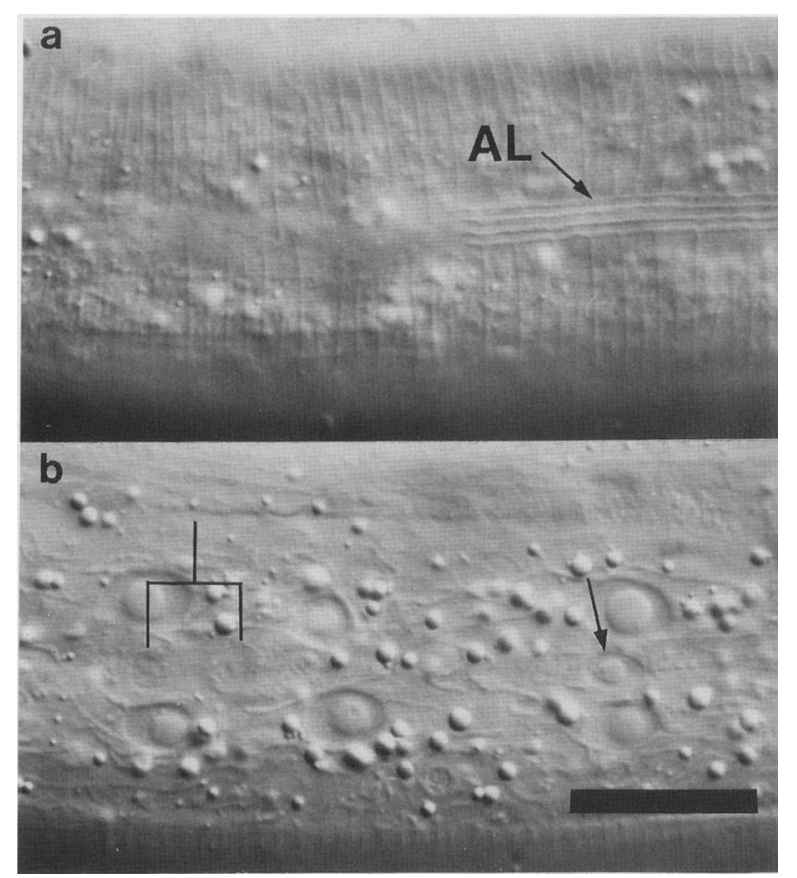

Figure 2. Photomicrograph showing the expression of both larval and adult seam cell fates at the L4 stage of a lin-14/n355 n679ts) hermaphrodite grown at $15^{\circ} \mathrm{C}$. The upper and lower panels show two planes of focus of the same animal taken a few seconds apart during L4 lethargus. The upper panel shows an area of the newly formed adult cuticle. This cuticle contains a segment of lateral alae (AL) and an adjacent area lacking alae. The bottom panel is in register with the top panel and shows the nuclei of seam cells underlying the cuticle. The seam cell on the left divided shortly before the photomicrograph was taken (daughter cell nuclei are indicated by the forked line); the cell on the right (nucleus indicated by the arrow) did not divide. Nomarski optics. Bar, $20 \mu \mathrm{m}$.

nDf19 show similar complementation patterns in combination with alleles of other classes. Specifically, when in trans to representative alleles of other phenotypic classes (e.g., $n 360$, class III; $n 536 n 838$, class IV), the class II allele $n 536 n 540$ and the deficiency $n D f 19$ are equivalent in that both fail to complement class III and class IV mutations for the class III and class IV defects, respectively (Table 3). For technical reasons, we have been unable to examine sufficient animals of the genotype $n 536 n 540 / n D f 19$ to compare them critically with n536 n540 homozygotes.

Although these observations are consistent with the five severe lin-14 recessive alleles being null alleles, it remains possible that these alleles reduce but do not eliminate lin-14 gene activity. True null alleles might cause additional defects, for example, lethality. Since we know that animals heterozygous for $n D f 19$ and a lin-14 semidominant allele [i.e., $n D f 19 /$ lin-14(sd)] are viable and less severely mutant than homozygous lin-14(sd) animals (see gene dosage experiments below), we have attempted to generate null alleles by screening among animals homozygous for a semidominant allele for partial revertants of the genotype lin-14(null)/lin-14(sd).
This experiment recovered one allele, $n 355 n 726$, which is one of the five severe class II alleles and is homozygous viable. The simplest interpretation of all of the results described in this section is that the severe class II alleles are null alleles. We consider them as such in the rest of this paper.

\section{The lin-14 locus controls two independently mutable stage-specific functions, lin-14a and lin-14b}

Certain heterochronic defects associated with reduction of lin-14 activity are mutationally separable. Although class II mutants display all the precocious defects listed in Table 1, class III mutants display strong precocious S3, S4 and precocious alae defects but no precocious S2 lineage defects, while the class IV mutants display a strong precocious $\mathrm{S} 2$ defect, a weak precocious alae defect, but no precocious S3 defect. Furthermore, class III mutations, e.g., lin-14(n360), which cause precocious alae but not precocious S2 lineage patterns, complement the class IV mutation lin-14(n536 n838) for the precocious S2 lineage defect (Table 3). These results suggest that the precocious S2 patterns and precocious alae defects are caused by loss of different lin-14 functions. We refer to these two independently mutable and complementing functions as lin-14a and lin-14b: Loss of lin-14a function $\left(\mathrm{a}^{-} \mid\right.$leads to precocious S2 lineage patterns, whereas loss of $l i n-14 b$ function $\left(b^{-}\right)$leads to precocious S3 and S4 patterns and precocious alae. Class II lin-14 recessive mutations are $\mathbf{a}^{-} \mathrm{b}^{-}$, while class III alleles (e.g., n360) are $\mathrm{a}^{+} \mathrm{b}^{-}$. Since the class IV allele (n536 n838) causes a strong precocious S2 lineage defect but only a very weak precocious alae defect, this allele is designated $a^{-} b^{1+1}$ (Table 3). Since the unique transformations in seam cell fates caused by loss of lin-14a and lin-14b affect cells at different stages, it appears that these two gene functions are stage-specific activities of lin-14 that control stage-specific choices of cell fates.

\section{Retarded defects appear to be caused by an elevation of lin-14 activity}

Since recessive lin-14 loss-of-function mutations reduce or eliminate lin-14 gene activity, and since lin-14 semidominant mutations cause transformations in seam cell fates opposite to those caused by recessive mutations (Ambros and Horvitz 1984; also Fig. 1), one possibility is that the semidominant mutations elevate lin-14 activity. To test this hypothesis, gene dosage experiments were performed to determine the effect of an increased level of wild-type lin-14 function on the severity of specific retarded seam cell fates (Table 4). In these experiments we compared the effects of a lin-14 wild-type allele to the effects of a presumptive null allele (n536 n540) in trans to lin-14 semidominant alleles. (No duplication of the lin-14 locus is available, so we have not tested the effects of two or more wild-type alleles in trans to semidominant alleles.) The semidominant alleles used were $n 536$ and $n 355$ n679. n536 causes retarded S1 lineage patterns (instead of the normal S2 pat- 
Table 2. Frequency of precocious seam cell fates in lin-14 recessive mutants

\begin{tabular}{|c|c|c|c|c|}
\hline Time & Genotype & $\begin{array}{l}\text { Percent alae } \\
\text { formation }\end{array}$ & $\begin{array}{l}\text { Percent S2 } \\
\text { lineage patterns }\end{array}$ & $n^{\mathbf{a}}$ \\
\hline \multirow{6}{*}{ L3 molt } & $+1+$ & 0 & & \\
\hline & $n D f 19 /+$ & 0 & & 120 \\
\hline & n179/n179 & 28 & & 172 \\
\hline & $n 179 /$ n536 n540 & 70 & & 96 \\
\hline & $n 179 / n D f 19$ & 75 & & 80 \\
\hline & n536 n540/n536 n540 & 100 & & 150 \\
\hline \multirow[t]{6}{*}{ Ll stage } & $+1+$ & & 0 & \\
\hline & $n D f 19 /+$ & & 0 & 30 \\
\hline & $n 179 / n 179$ & & 28 & 43 \\
\hline & $n 179 / n 536$ n540 & & 67 & 49 \\
\hline & $n 179 / n D f 19$ & & 61 & 46 \\
\hline & n536 n540/n536 n540 & & 93 & 43 \\
\hline
\end{tabular}

The expressivity of individual lin-14 heterochronic phenotypes was measured by determining the frequency of specific heterochronic transformations in seam cell fates at the stages indicated as described in Materials and methods. For the most part, the variability in the cell lineage patterns of these mutants seems to be discrete and binary; i.e., each seam cell at a particular stage expressed one of two specific alternative fates-at the L1 stage, seam cells either generated an S1 pattern or an S2 pattern; at the L3 molt, each cell either formed adult lateral alae or divided. Occasionally, we observed a cell division pattern different from any wild-type pattern. We have not considered those rare abnormal patterns here. Seam cell fates were determined for cells derived from blast cells V1-V4 and V6, which normally generate identical lineages (Sulston and Horvitz 1977).

a Total number of seam cells for which fates were scored. In the experiments shown here and in Table 3, the number of seam cells scored per animal varied from six to greater than 20, depending upon whether one or both sides of the animal were scored and upon developmental factors (stage, cell lineage reiterations) affecting number of seam cells. Strains were constructed and fates were scored at $20^{\circ} \mathrm{C}$ using anatomical screens as described in Materials and methods. $n D f 19$ is a deficiency of the lin-14 locus (Ambros and Horvitz 1984). lin-14(n179) animals maintained at $20^{\circ} \mathrm{C}$ display a relatively mild class II phenotype, intermediate between the severe phenotype of $\operatorname{lin}-14(\mathrm{n} 179)$ animals at $25^{\circ} \mathrm{C}$ (approximately $100 \%$ alae formation by seam cells at the $\mathrm{L} 3$ molt) and the essentially wild-type development displayed at $15^{\circ} \mathrm{C}$.

terns) at the L2 stage and retarded S3 or S4 lineage patterns (instead of the normal adult alae formation) at the L4 molt (Table 1); n355 n679 causes retarded S3 or S4 lineage patterns at $15^{\circ} \mathrm{C}$ (Figs. 1, 2, and 3). [lin-14(n355) was not used in these experiments because of the difficulty of strain constructions with this highly penetrant allele.]

The results of these gene dosage experiments are con- sistent with the hypothesis that the lin-14 semidominant mutations lead to an elevation of wild-type lin-14 activity. The principal evidence supporting this hypothesis is that retarded defects are more severe when a wild-type allele of $l i n-14$ is in trans to a semidominant lin-14 allele than when a null allele is in trans to the same semidominant allele. For example, hermaphrodites carrying one $n 355$ n679 allele and one copy of the

Table 3. Complementation between lin-14 recessive alleles for precocious seam cell fates

\begin{tabular}{|c|c|c|c|c|c|c|c|c|}
\hline & & & \multicolumn{2}{|c|}{$\begin{array}{c}\text { II } \\
\text { n536 n540 } \\
a^{-} b^{-}\end{array}$} & \multicolumn{2}{|c|}{$\begin{array}{c}\text { III } \\
n 360 \\
a^{+} b^{-}\end{array}$} & \multicolumn{2}{|c|}{$\begin{array}{c}\text { IV } \\
n 536 n 838 \\
a^{-} b^{1+1}\end{array}$} \\
\hline & & & S2 & alae & $\mathrm{S} 2$ & alae & $S 2$ & alae \\
\hline & $n D f 19$ & $a^{-} b^{-}$ & $\star \star \star a$ & $\star \star *$ & $w t^{b}$ & $\star \star \star *$ & $\star \star \star \star$ & $* *$ \\
\hline II & n536 n540 & $a^{-} b^{-}$ & $\star \star \star$ & $\star \star \star$ & wt & $\star \star \star *$ & $\star \star \star *$ & ** \\
\hline III & $n 360$ & $a^{+} b^{-}$ & & & $w t$ & 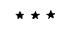 & wt & $\star \star$ \\
\hline IV & $n 536$ n838 & $a^{-} b^{(+)}$ & & & & & $\star \star \star \star$ & $\star$ \\
\hline
\end{tabular}

Alleles representing each of the three classes of recessive lin-14 mutants (II, III, IV) were tested in trans to each other and to $n D f 19$, a deficiency of the lin-14 locus (Ambros and Horvitz 1984). Strains were constructed and phenotypes were scored as described in Materials and methods. For each heteroallelic combination, two heterochronic defects, precocious S2 patterns (S2) and precocious adult alae formation (alae), were scored by screening animals of each genotype for specific anatomical abnormalities that reflect those defects.

${ }^{*}\left(,^{\star *},{ }^{\star *}\right)$ The alleles failed to complement and expressed the defect with a corresponding severity as described in the legend to Table 1.

b (wt) The defect was not observed (i.e., the alleles complemented).

The observed complementation pattern is postulated to reflect two independently mutable lin-14 functions, lin-14a and lin-14b (see text, Fig. 9). The interpretation of each allele is shown in brackets; $n D f 19$ and $n 536 n 540$ eliminate both activities [a- $\left.\mathrm{b}^{-}\right], n 360$ causes loss of only lin-14b $\left[\mathrm{a}^{+} \mathrm{b}^{-}\right]$, and $n 536 n 838$ leads primarily to a loss of lin-14a and a slight loss of lin-14b [a- $\left.\mathrm{b}^{1+1}\right]$. All experiments were performed at $20^{\circ} \mathrm{C}$. 
Table 4. Gene dosage effects on the frequency of stage-specific seam cell fates in lin-14 semidominant mutants

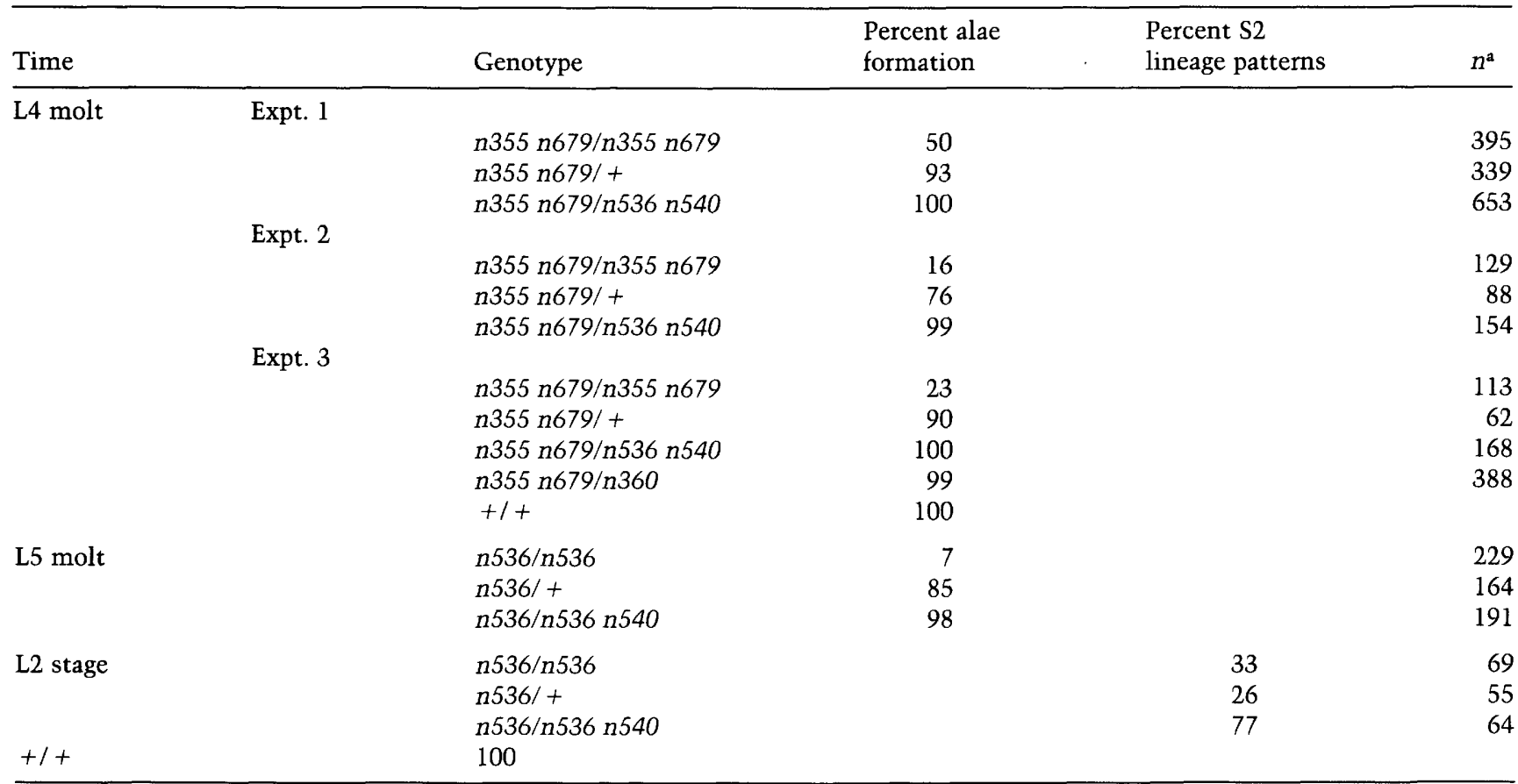

The expressivity of lin-14 heterochronic defects was determined by measuring the frequency of specific transformations in seam cell fates at specific developmental stages. The frequency of seam cells undergoing cell division (presumably expressing S3 or S4 lineage patterns) was determined by quantifying the number of seam cells expressing the alternative fate-adult alae formation. Adult alae formation was determined as described in Materials and methods. The frequency of seam cells expressing retarded S1 lineage patterns was determined by quantifying the number of seam cells expressing the alternative fate-S2 lineage patterns. Lineage patterns were characterized using anatomical screens or by continual observation, as described previously by Sulston and Horvitz (1977). In the L2 stage of these mutants, most seam cells expressed either S2 lineage patterns or S1 lineage patterns. Occasionally, cell division patterns at this stage differed from all lineage patterns that occur in the wild type. We have not considered these rare abnormal patterns here. Experiments with $n 536$ were performed at $20^{\circ} \mathrm{C}$; those with $\operatorname{lin}-14(n 355 n 679 t s)$ were performed at $15^{\circ} \mathrm{C}$. Experiments 1,2 , and 3 were performed on separate occasions using different protocols (see Materials and methods). We suspect that the absolute frequency of adult alae formation for animals carrying lin-14(n355 n679ts) is very sensitive to small variations in temperature between experiments. Within each experiment, care was taken to insure that all animals were maintained at the same temperature.

a $(n)$ Total number of seam cells scored.

wild-type lin-14 locus, (n355 n679/ +), had a stronger retarded phenotype (absence of alae formation by seam cells at the L4 molt) than hermaphrodites carrying one n355 n679 allele and a null allele of $\operatorname{lin}-14$ (n355 n679/ $n 536$ n540) (Fig. 3; Table 4). Animals with two semidominant alleles (n355 n679/ n355 n679) had a still stronger retarded phenotype (Fig. 3; Table 4). Thus, the retarded phenotype of animals carrying one semidominant allele can be increased in severity by the addition of a wild-type allele and, to a greater degree, by a second semidominant allele. Similar results were obtained by comparing $n 536 /$ null animals, $n 536 /+$ animals, and $n 536 / n 536$ animals for the penetrance of a retarded phenotype (absence of alae formation by seam cells) at a supernumerary (L5) molt and for another retarded defect (expression of S1 lineage patterns instead of S2 patterns) at the L2 stage (Table 4).

These observations indicate that the effects of one wild-type allele in trans to a semidominant allele are qualitatively similar to (although quantitatively less severe than) the effects of two semidominant alleles. It ap- pears that the wild-type allele of $\operatorname{lin}-14$ results in a certain amount of gene function and the lin-14 semidominant alleles result in relatively higher amounts of the same function. Although other interpretations are possible, we feel that the above proposal - that 1 in-14 semidominant alleles elevate lin-14 activity-is the simplest model consistent with both the results of these genedosage experiments and with the observation that lin-14 semidominant alleles cause defects opposite to those caused by lin-14 null alleles.

A semidominant mutation might act in cis to cause elevated lin-14 function, for example, by inactivating a negative-regulatory region or by activating a promoter. If our semidominant lin-14 alleles act only in cis, our finding that the retarded phenotype of animals carrying one semidominant allele can be increased in severity by the addition of a wild-type allele in trans suggests that the wild-type allele results in a "low" but non-zero level of activity for the affected cells and that the semidominant mutation results in a "high" level of activity. In this case, the more severe phenotype of $l i n-14(s d) /+$ an- 

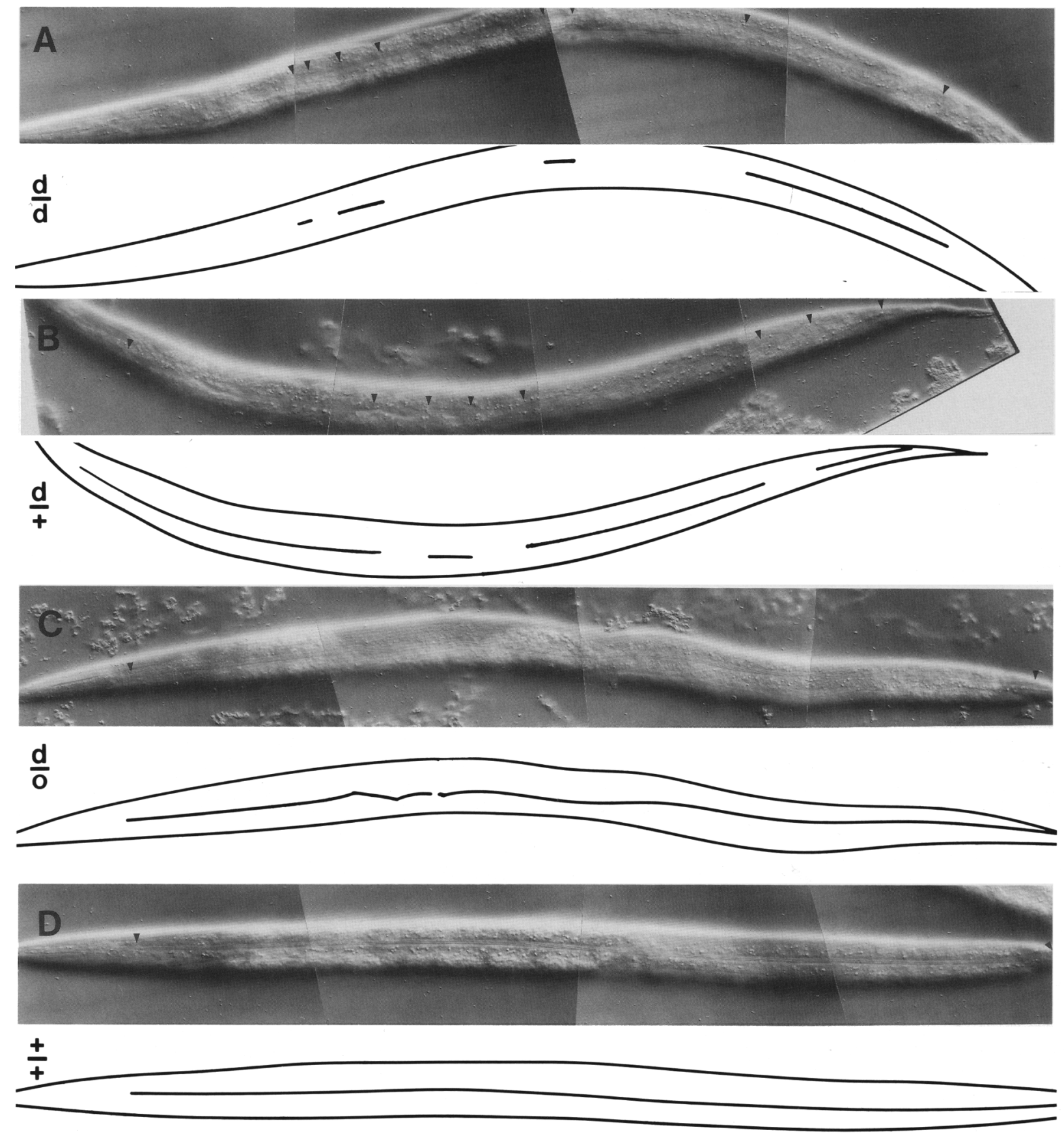

Figure 3. The effect of dosage of the wild-type and a semidominant lin-14 allele on the frequency of seam cell fates at the L4 molt. These photomicrographs show left lateral views of the cuticle of representative animals of four different lin-14 genotypes. The mutation $n 355 n 679$ was chosen to illustrate the effects of dosage because of the broad range of severity observed with the different genotypes carrying this allele. To facilitate examination of these photomicrographs, the end points of lateral alae segments are indicated by arrowheads on the photomicrographs, and each lateral alae segment is shown as a dark line in the drawing below each photomicrograph. In each animal, seam cells underlying areas of cuticle lacking alae underwent cell division at the L4 molt, while cells underlying segments of lateral alae did not divide (see Fig. 2). Nomarski optics. Bar, $100 \mu \mathrm{m}$. (A) lin-14(n355 n679)/lin-14/n355 n679) animal (genotype designated here as $\mathrm{d} / \mathrm{d}$ for clarity) in which eight of 23 seam cells on the left side formed lateral alae at this stage. $(B) \operatorname{lin}-14(\mathrm{n} 355 \mathrm{n679}) /+$ animal $(\mathrm{d} /+)$ in which 10 of 14 seam cells on the left side formed alae at this stage. $(C)$ lin-14(n355 n679)/lin-14(n536 n540) animal (d/0; i.e., d/"null") in which 11 of 11 seam cells on the left side formed lateral alae at the L4 stage. (D) Wild-type animal $(+/+)$ in which 11 of 11 seam cells on the left side formed lateral alae at this stage. Animals were maintained, staged, and screened as in Table 4 . Animals were grown at $15^{\circ} \mathrm{C}$. Some of these animals had greater than the normal number of seam cells due to reiterations of the S2 lineage pattern (see Fig. 1). 
imals would result from the summation of these two levels. Alternatively, there could be a simultaneous cis and trans action of the lin-14 semidominant alleles: The wild-type lin-14 allele may normally be completely inactive ("off") for the affected cells and the semidominant allele may cause the inappropriate activation both of itself (in cis) and of the wild-type allele (in trans). Although the hypothesis of cis action may seem simpler, there is precedent for such trans activation (e.g., the Drosophila sex lethal gene, $S x l$; Cline 1984). Since our data do not distinguish between these two possibilities, we will refer to alternative levels of lin-14 function as "high" and "low" with the understanding that these terms encompass "on" and "off".

The complex class $V$ phenotype is caused by the loss of lin-14 activity for certain cells and the elevation of lin14 activity for other cells

As described above, the allele lin-14(n355 n679) is semidominant at $15^{\circ} \mathrm{C}$, causing the retarded expression of certain cell lineage patterns and supernumerary molts. However, lin-14(n355 n679) animals also express precocious defects. For example, a single lineage containing both the precocious and the retarded expression of S2 lineage patterns in a lin-14(n355 n679) animal at $15^{\circ} \mathrm{C}$ is shown in Figure 1f. Since the expression of precocious S2 lineages is caused by the loss of lin-14 activity, and the expression of the retarded S2 lineages is caused by the elevation of lin-14 activity, this complex phenotype appears to result from reduced lin-14 for cells in the L1 and elevated lin-14 for cells in the L3. Furthermore, for animals carrying either lin-14(n355 n679) or the other class $\mathrm{V}$ allele, lin-14(n536 n538), we have observed precocious adult alae formation by seam cells at the L3 molt followed by lack of adult formation by seam cells at the L4 molt in the same animal (although not in the same lineage). The lack of adult alae seems to be caused by an elevation in lin-14b, since the mutation $n 360$, which reduces lin-14b but not lin-14a activity, almost completely prevents the expression of at least one retarded defect when in trans to $n 355 n 679$ (Table 4). Thus, it appears that in lin-14(n355 n679) or lin-14(n536 n538) animals, reduced lin-14b activity for cells at the L3 molt results in the precocious formation of adult alae and elevated lin-14b activity for cells at the $\mathrm{L} 4$ molt results in the retarded expression of S3 or S4 lineage patterns. These observations suggest that $\operatorname{lin}-14$ (n355 n679) and lin-14(n536 n538) are partial revertants of lin-14(n355) and lin-14(n536), respectively, and that the mixture of retarded and precocious defects reflects an incompletely penetrant recessive phenotype conferred by the mutations $n 679$ or $n 538$. Thus, we propose that in class $\mathrm{V}$ mutants, the $n 355$ and $n 536$ mutations cause elevated lin-14 levels for certain cells, while other cells in the same animal variably experience a loss of $\operatorname{lin}-14$ activity caused by the $n 679$ or $n 538$ mutations.

lin-14a and lin-14b act at different times during development

lin-14 mutations affect the fates of lateral hypodermal cells at all postembryonic stages (Fig. 1; Table 1). As de- scribed above, lin-14a, which normally controls cell fates expressed during the L1 stage, is mutationally separable from lin-14b, which normally controls cell fates expressed at later stages. When during development do these lin-14 activities function? For example, lin-14a and lin-14b might both function at the same time early during development to specify subsequent cell fates. Alternatively, lin-14a and lin-14b might act at different times.

The removal or elevation of lin-14 activities selectively at specific stages of development would reveal the times during development that altered levels of gene activity can affect the fates of specific cells. For this reason, we have performed temperature-shift experiments using lin-14 alleles that are temperature sensitive (ts) either for the elevation of gene activity or for the loss of gene activity.

ts period for loss of lin-14a activity As described above, recessive alleles of classes II and IV cause the loss of lin-14a activity and lead to the precocious expression of S2 lineage patterns by cells in the L1 stage. For example, at $25^{\circ} \mathrm{C}$, lateral hypodermal seam cells of lin-14(n179ts) animals express S2 lineage patterns during the L1. This allele is ts, since at $15^{\circ} \mathrm{C}$ seam cells express their normal fates. Temperature-shift experiments using lin-14(n179ts) animals showed that the ts period for the production of precocious S2 lineage patterns by seam cells lies in an interval that begins shortly after hatching and ends just before the first division of those cells (Fig. 4). Animals must be maintained at $15^{\circ} \mathrm{C}$ during the ts period for seam cells in the $\mathrm{L} 1$ to express their normal fates (S1 patterns), suggesting that lin-14a activity is required during that interval for the expression of S1 lineage patterns. Loss of 1 in-14a activity during all or part of the ts period can cause these cells to express normally later fates (S2 patterns).

ts period for loss of lin-14b activity lin-14(n179ts) is also temperature sensitive for defects caused by loss of lin-14b, i.e., the precocious expression of S3 lineage patterns in the L2 and the precocious formation of adult alae at the L3 molt. Temperature-shift experiments were performed using animals of genotypes lin-14(n179ts) and lin-14(n179ts)/lin-14(n360). This latter strain was constructed to obtain an $\mathbf{a}^{+} \mathbf{b}^{-}$ts strain so that the ts period for lin-14b could be determined independently of lin-14a. The ts period for precocious expression of S3 cell lineage patterns by the seam cells of lin-14(n179ts)/ lin-14(n360) animals is an interval during the mid-late Ll stage distinct from the lin-14a ts period (Fig. 5a). These results indicate that $\operatorname{lin}-14 a$ and $\operatorname{lin}-14 b$ are required at separate times during development for seam cells in the L1 and L2, respectively, to express their normal fates as opposed to later fates. The ts period for the precocious expression of adult alae formation at the L3 molt in lin-14(n179) animals (Fig. 5b) appears to be approximately coincident with the ts period for precocious S3 lineages (Fig. 5a). This finding is consistent 
$\underline{\operatorname{lin}-14(\mathrm{n} 179)}$ L 1 stage $S 1$ vs $S 2$

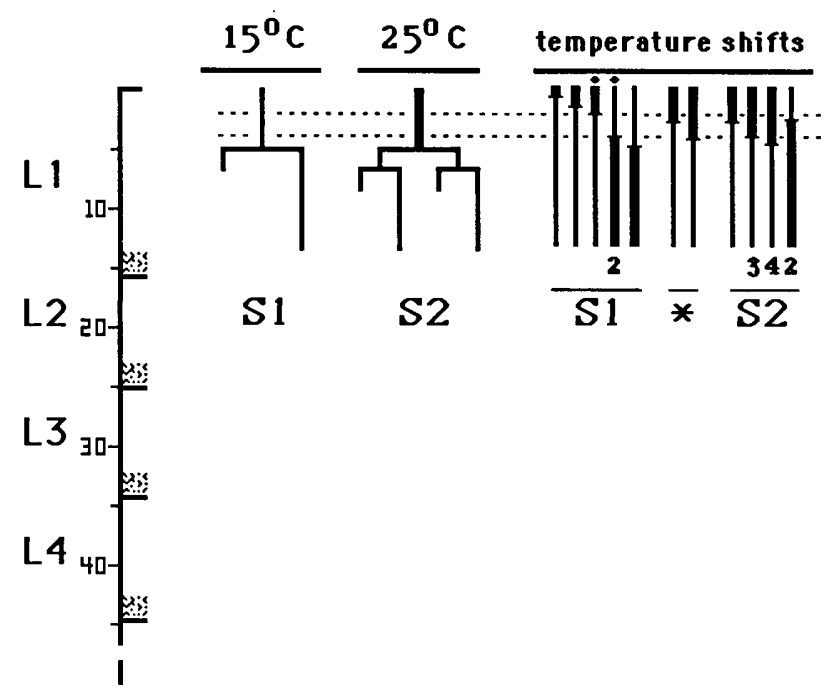

with the observation that mutations that alter lin-14b activity coordinately affect these two events (see above). However, in mutants carrying certain lin-14 alleles (particularly $n 727$ ) we have observed the precocious expression of adult alae in cell lineages in which S3 lineage patterns were expressed at the normal time. This result indicates that although lin-14b activity affects both the timing of S3 lineage patterns and the timing of adult alae formation, these two events can be altered independently.

ts period for elevation of lin-14b activity It is likely that the retarded defects of $\operatorname{lin}-14(n 355$ n679) animals are caused by elevation of lin-14b. For example, the mutation, lin-14(n360), which reduces lin-14b activity, alleviates the retarded phenotype when placed in trans to n355n679 (Table 4). Thus, temperature-shift experiments between $15^{\circ} \mathrm{C}$ and $20^{\circ} \mathrm{C}$ using lin-14(n355 n679ts) animals revealed the ts periods for defects caused by the elevation of lin-14b activity. The ts periods for both of the retarded defects scored, retarded S2 lineage patterns instead of S3 patterns at the L3 stage and retarded cell division instead of adult alae formation at the $\mathrm{L} 4 \mathrm{molt}$, are approximately coincident intervals during the L2 (Fig. 6). This ts period represents an interval during which the wild-type level of 1 in-14b activity allows cells at the L3 stage (Fig. 6a) and at the L4 molt (Fig. 6b) to express their normal fates; however, if $l i n-14 b$ levels are higher than the wild-type level, these cells can express fates that normally occur earlier in development.

\section{Discussion}

The role of lin-14 in wild-type development

Our developmental and gene-dosage studies indicate that lin-14 determines the stage-specific expression of
Figure 4. Temperature-sensitive (ts) period for loss of lin-14a activity. For the experiments described in this figure, as well as in Figs. 5 and 6, animals were maintained, developmental stages were determined, and individual animals were shifted from one temperature to another as described in Materials and methods. The results of these experiments were used to estimate the ts period for expression of the indicated alternative seam cell fates at the specified stage of development. Because of the relatively small numbers of animals used in these experiments, and because we wished to assess possible overlaps between the ts periods for different defects, in each of these figures, we have used dotted lines to mark the beginning and end of an interval that, based on these data, includes the entire ts period. In this figure, and in Figs. 5 and 6, the interval containing the ts period was derived from the data as follows: The beginning of the interval (the first dotted line in each figure) indicates the time of the latest of a set of downshifts (from high to low temperature) that all resulted in a phenotype like that of animals maintained at the lower temperature (by both quantitative and qualitative criteria as described below); i.e., this is the latest time before which our data show no requirement for wild-type gene activity. The end of the interval (the second dotted line) indicates the time of the earliest of a set of upshifts (from low to high temperature) that all resulted in a phenotype like that of animals maintained at the lower temperature; i.e., this is the earliest time after which our data show no requirement for wild-type gene activity. Each vertical bar represents a single shifted animal except in cases in which multiple animals were shifted at the same stage; the number of such animals is shown below the vertical bar. The specific shifts that define the beginning and end of the ts period (see above) are indicated by a diamond above the bar. Thin segments of each vertical bar show the portion of development when the animal was at $15^{\circ} \mathrm{C}$, and thick segments indicate the portion of development at $25^{\circ} \mathrm{C}$. The time of the shift was determined by examining each animal for the occurrence of certain stage-specific developmental events, as described in Materials and methods, and is shown with respect to the time scale at left, calibrated in hours after hatching at $20^{\circ} \mathrm{C}$. Animals were shifted either at the time of one of these events or between two of these events. In these mutants, seam cells at a specific stage express one of two alternative fates, either the normal fate or a heterochronic fate. The portion of seam cells that express one or the other of these alternative fates depends upon temperature: As indicated in these figures, for animals maintained at one temperature, most cells express one of these fates (and a minority express the alternative), while most cells express the opposite fate at the other temperature. In temperature-shifted animals, the portion of seam cells that expressed either the "high temperature" fate or the "low temperature" fate depended upon the time of the shift. The phenotype of each shifted animal was classified according to the portion of its seam cells that expressed one of these alternative fates as opposed to the other at the stage in question: If a sufficiently large portion (see below) of seam cells expressed one fate, the phenotype of that animal was classified as equivalent to animals maintained at the corresponding temperature. If the portion of seam cells expressing either of the two alternative fates was within an intermediate range (as specified below and in the legends to Figs. 5 and 6 for each mutant) the phenotype of that animal was classified as "intermediate." In this figure, shifted animals were scored as equivalent to $15^{\circ} \mathrm{C}$-grown animals if $80-100 \%$ of the seam cells expressed $\mathrm{S} 1$ patterns, like $25^{\circ} \mathrm{C}$-grown animals if $0-33 \%$ expressed $\mathrm{S} 1$ patterns, and of intermediate $\left({ }^{*}\right)$ phenotype if $34-79 \%$ expressed S1 patterns. (More than $95 \%$ of the seam cells of L1 lin-14(n179) animals express $\mathrm{S} 1$ lineage patterns at $15^{\circ} \mathrm{C}$, and fewer than $20 \%$ express Sl lineage patterns at $25^{\circ} \mathrm{C}$. 
a. $\quad$ lin-14(n $179 / \mathrm{n} 360)$ L2 stage S2 vs $S 3$

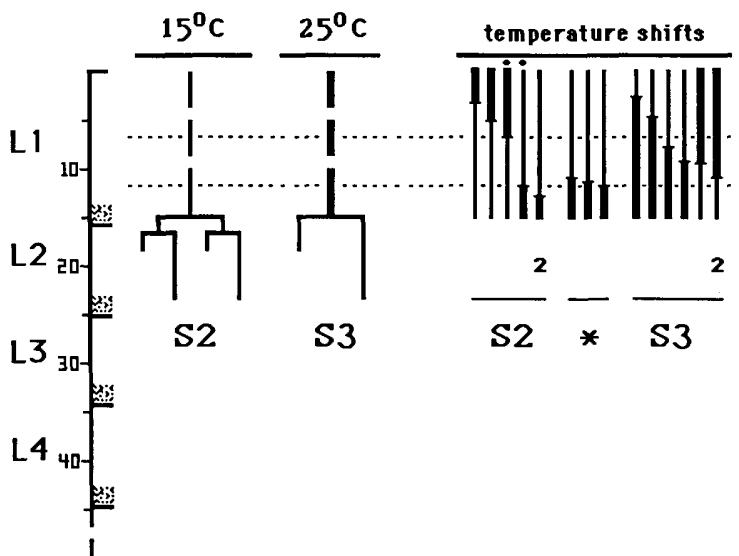

b. lin-14(n 179) L.3 molt divide vs form alae

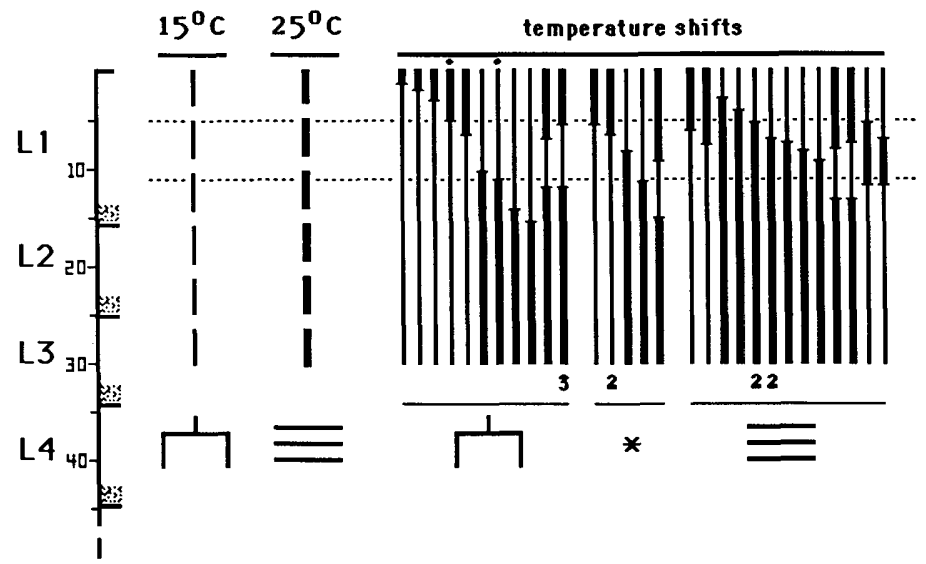

Figure 5. Temperature-sensitive (ts) period for loss of lin-14b activity. Experiments were performed and are depicted as described in the legend to Fig. 4 and in Materials and methods. The two vertical bars at the left in each panel are broken to denote that cell divisions may have occurred at earlier stages but are not shown explicitly. Only the fates expressed at the indicated stages were scored in each experiment. In this figure, and in Fig. 6, certain animals were shifted and then later returned to the original temperature. The times and durations of these "pulse shifts" are shown. The phenotypes of pulse-shifted animals were generally consistent with the ts periods derived from singly shifted animals. $(a)$ Shifted animals were scored as equivalent to $15^{\circ} \mathrm{C}$-grown animals if $0-30 \%$ of the seam cells expressed S3 patterns, like $25^{\circ} \mathrm{C}$-grown animals if $67-100 \%$ expressed S3 patterns and of intermediate phenotype if 31-66\% expressed S3 patterns. [Less than 10\% of the seam cells of L2-stage lin-14(n179/lin-14(n360) animals precociously express S3 patterns at $15^{\circ} \mathrm{C}$, while more than $80 \%$ express S3 patterns at $25^{\circ} \mathrm{C}$.] Presumably because of temperature-sensitive lin-14b activity, lin-14 (n179) fails to complement the lin-14b mutation $n 360$ at $25^{\circ} \mathrm{C}$. In lin-14(n179)/lin-14(n360) animals (as in lin-14(n360) animals; see Fig. 1), L1-stage lineage patterns are normal at both temperatures, allowing identification of the ts period of $\operatorname{lin}-14(\mathrm{n} 179)$ for L2 fates alone. (b) Shifted animals were scored as equivalent to $15^{\circ} \mathrm{C}$-grown animals if $0-20 \%$ of their seam cells formed lateral alae, like $25^{\circ} \mathrm{C}$-grown animals if $70-100 \%$ formed lateral alae, and of intermediate phenotype if $21-69 \%$ formed lateral alae. [Less than $10 \%$ of the seam cells at the $\mathrm{L} 3$ molt of $\operatorname{lin}-14(\mathrm{n} 179)$ animals from alae at $15^{\circ} \mathrm{C}$, and about $95 \%$ form alae at $25^{\circ} \mathrm{C}$.] In these mutants, all seam cells that do not form alae at a given stage appear to divide instead (see Fig. 2 and text).

a. 1 in-14(n355 n679) L3 stage S2 vs S3

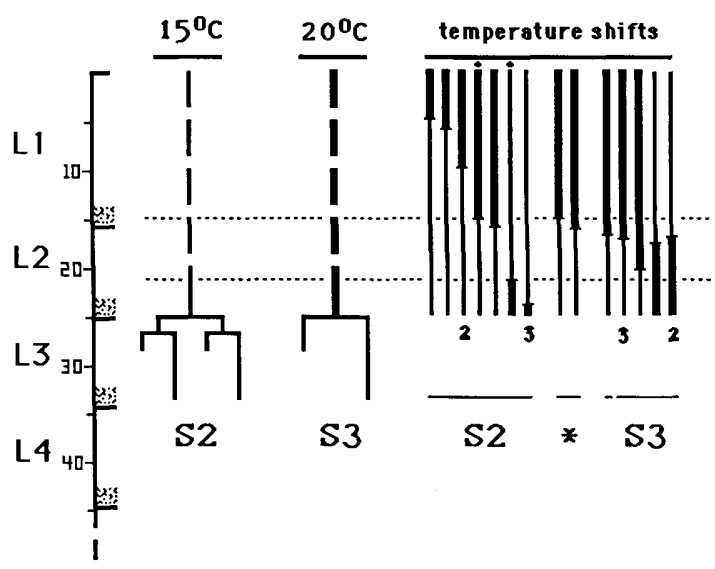

b. 1 in-14(n355 n679) L4 molt divide vs form alae

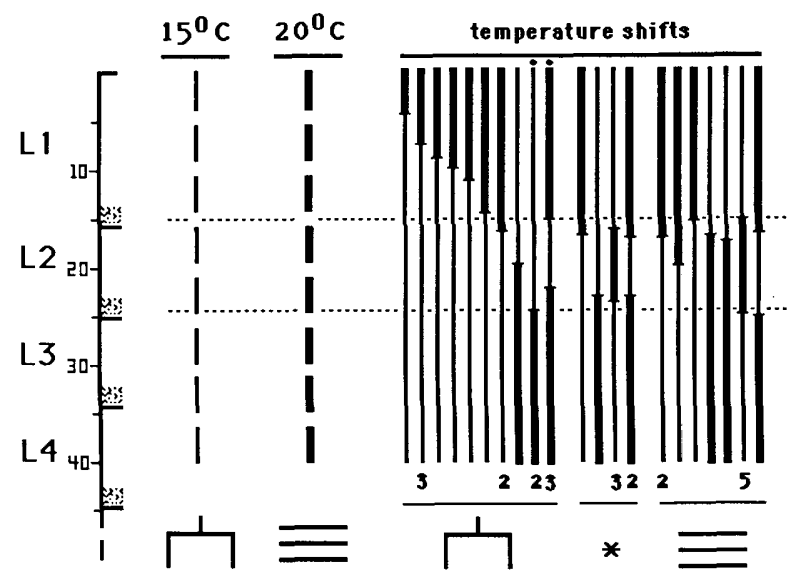

Figure 6. Temperature-sensitive (ts) period for elevation of lin-14b activity. Experiments were performed as described in the legends to Figs. 4 and 5 and in Materials and methods. (a) Shifted animals were scored as equivalent to $15^{\circ} \mathrm{C}$-grown animals if more than $50 \%$ of the seam cells expressed S2 patterns, equivalent to $20^{\circ} \mathrm{C}$-grown animals if less than $20 \%$ expressed S2 patterns, and of intermediate phenotype if $21-49 \%$ expressed S2 patterns. [Approximately $55 \%$ of the seam cells of L2-molt (or early L3) lin-14(n355 n679) animals express retarded S2 lineage patterns at $15^{\circ} \mathrm{C}$ and less than $10 \%$ express retarded S2 patterns at $20^{\circ} \mathrm{C}$.) $(b)$ Shifted animals were scored as equivalent to $15^{\circ} \mathrm{C}$-grown animals if $0-35 \%$ of seam cells formed lateral alae, as equivalent to $20^{\circ} \mathrm{C}$-grown animals if $75-100 \%$ formed lateral alae, and of intermediate phenotype if $36-74 \%$ formed lateral alae. [Approximately $20 \%$ of the seam cells of L4-molt lin-14(n355 n679) animals formed adult lateral alae at $15^{\circ} \mathrm{C}$, while greater than $90 \%$ formed lateral alae at $20^{\circ} \mathrm{C}$.] 
seam cell fates. Mutations that eliminate lin-14 activity cause cells to express fates normally expressed by other cells at later stages, and mutations that elevate lin-14 activity cause cells to express fates normally expressed by other cells at earlier stages. In other words, high levels of lin-14 activity result in earlier fates and low levels result in later fates (Fig. 7a). These observations suggest that during normal development lin-14 activity may be temporally graded, high at early times and low at late times (Fig. 7b). According to this model, mutations that elevate lin-14 activity cause it to be inappropriately high at late times, resulting in the reiteration of earlier fates, and mutations that eliminate lin-14 activity cause it to be inappropriately low at early times, resulting in the precocious expression of later fates.

Our temperature-shift experiments support this model. By reducing or elevating lin-14 activity at different times during development, we have shown that this gene functions at a number of distinct times. For example, the level of lin-14 activity during the late-L1 stage determines which lineage patterns are expressed during the L2 stage (Fig. 5), and the level of lin-14 activity during the L2 stage determines which lineage patterns are expressed during the L3 stage (Fig. 6). At both times, a high level of lin-14 activity results in the normally earlier cell fates (S2 lineage patterns) and a low level results in the normally later cell fates (S3 patterns). These results suggest that in the wild type during the late-L1 stage lin-14 activity is high, causing the expression of S2 patterns in the L2 stage, and that during the L2 stage lin-14 activity is low, causing the expression of S3 patterns in the L3 stage.
We can extend our general model for lin-14 action (Fig. 7) to account for the details of wild-type development, the defects caused by the five phenotypic classes of lin-14 alleles, and the ts periods of the temperature-sensitive alleles. Our complementation and temperatureshift studies have defined two distinct lin-14 activities, lin-14a and lin-14b, which act at different times during development. We propose that the loss of $\operatorname{lin}-14 a$ activity leads to precocious S2 lineage patterns, and the loss of lin-14b activity leads to precocious expression of the sequence of events consisting of S3 patterns, S4 patterns, and adult alae formation (S3/S4/alae); similarly, increased lin-14a activity leads to retarded S1 patterns, and increased lin-14b activity leads to retarded S2 patterns. According to this model, the different classes of Iin-14 alleles appear to affect these two activities in different ways (Fig. 8): Wild-type animals display normal development and are normal for both activities $\left(\mathrm{a}^{+} \mathrm{b}^{+}\right)$; class I mutants are retarded for both S1 and S2 patterns and are $\mathbf{a}^{++} \mathbf{b}^{++}$; class II mutants are precocious for both $\mathrm{S} 2$ patterns and S3/S4/alae and are $\mathrm{a}^{-} \mathrm{b}^{-}$; class III mutants are precocious for S3/S4/alae only and are $a^{+} b^{-}$; the class IV mutant is precocious for S2 patterns only and is $a^{-} b^{+}$; and class $V$ mutants are precocious for $S 2$ patterns and retarded for S2 patterns and are $a^{-} b^{++}$. (Since class $\mathrm{V}$ mutants were isolated by mutagenizing class I mutants, their phenotype can be regarded as resulting from a class IV mutation in a class I background.)

We consider two models that can account for the existence of these two lin-14 activities (Figs. 8 and 9). First, lin-14 might encode two distinct gene products for two functionally distinct domains of a single gene product)

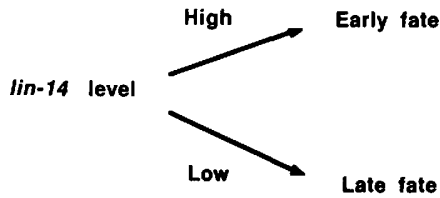

b.

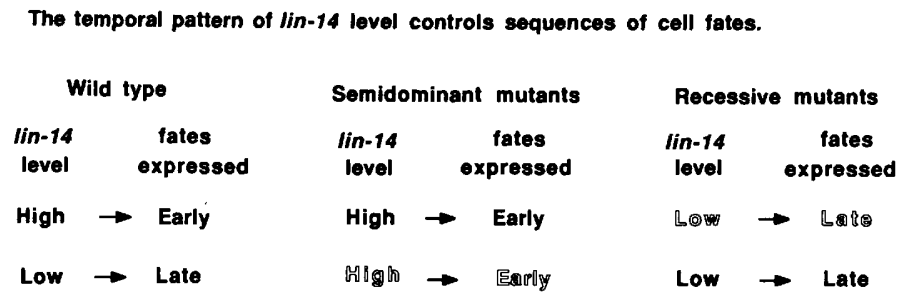

Figure 7. (a) A model for how lin-14 selects the fates of lateral hypodermal seam cells. Certain seam cells have two developmental potentials - expression of an "early" fate or expression of a "late" fate. The level of lin-14 function during a critical period selects which of those fates is expressed by each cell. A high level of activity selects an early fate, and a low level selects a late fate. $(b)$ A model for how lin-14 controls the stage-specific expression of cell fates in the lateral hypodermal lineages. In the wild-type, lin-14 level changes from high early in development to low at later stages, causing the expression of first early then late fates. In lin-14 semidominant mutants, lin-14 activity remains inappropriately high at later stages, resulting in the reiteration of early fates. In lin-14 recessive mutants, lin-14 activity is low throughout development resulting in the precocious expression of late fates. Abnormal levels of activity at specified stages and the resulting heterochronic cell fates are shown with open-faced type. 
a. Model 1: two products, two levels of each.

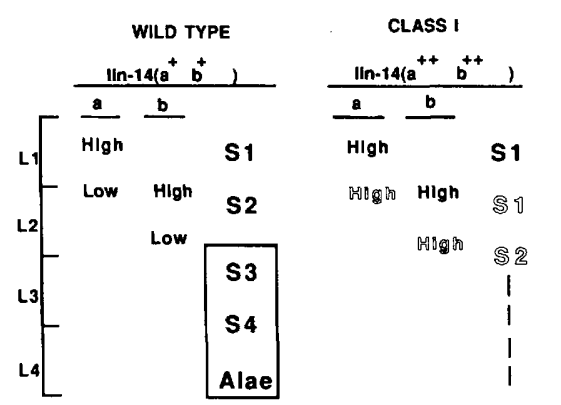

b. Model 2: one product, three levels.

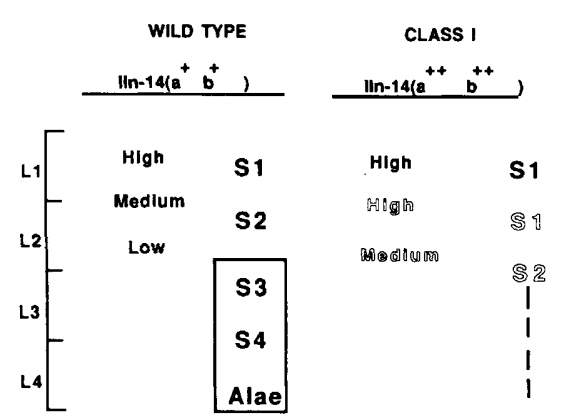

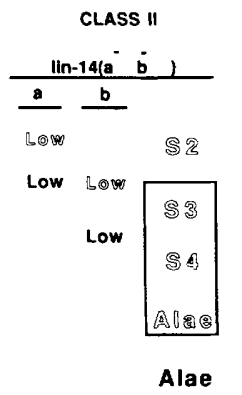
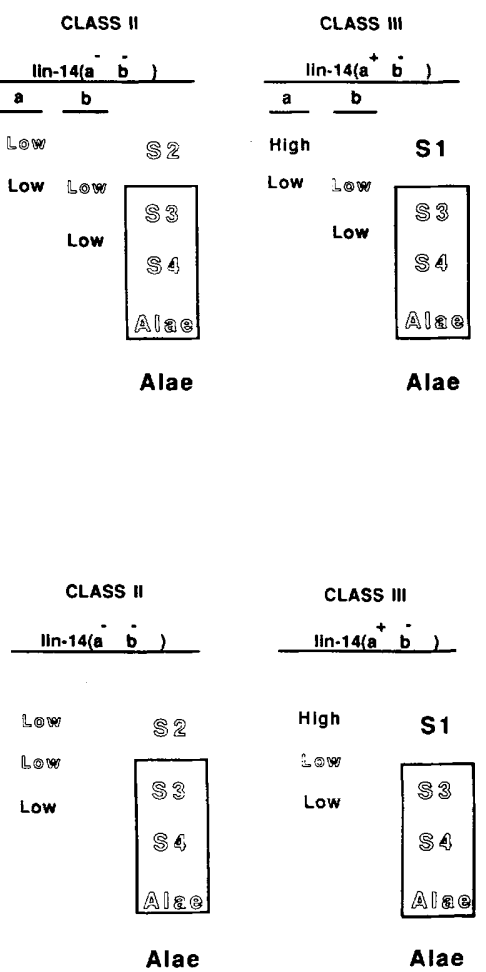
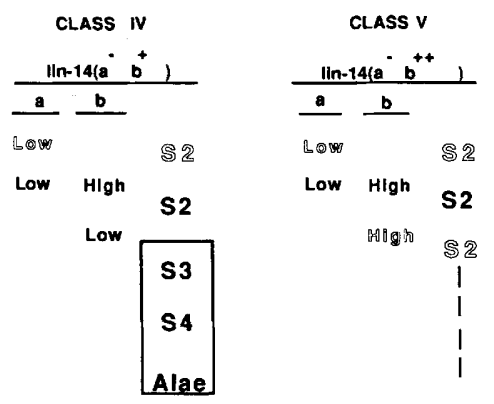

Figure 8. Two alternative models for how lin-14 acts stage-specifically to control choices of cell fates. Stage-specific lin-14 activities are defined as $\operatorname{lin}-14 a$ and $\operatorname{lin}-14 b$ (see text). For each class of mutant, a typical temporal pattern of developmental events is shown and our interpretation of the genotypes of lin-14a and lin-14b activities are indicated by superscripts: $|-|$ loss of activity; $|+|$ wild-type level of activity; $(++)$ elevated level of activity (see text). For simplicity, each mutant class is interpreted as having a genotype that corresponds to this typical developmental pattern. For example, the genotypes of class IV and class $\mathrm{V}$ mutants are shown as $\mathrm{a}^{-} \mathrm{b}^{+}$and $\mathrm{a}^{-} \mathrm{b}^{++}$, respectively, because according to our model, those levels of lin-14 activities result in the specific patterns shown; however, other lineage patterns can occur in individuals of those particular mutant classes (see Table 1). (a) The first model proposes that lin-14a and lin-14b reflect the activities of two functionally distinct lin-14 products that are independently regulated. Shown here are the levels of $l i n-14 a$ and $l i n-14 b$ at each stage of development and the resulting temporal patterns of lateral hypodermal cell fates expressed during wild-type development and during development of the five classes of lin-14 mutants described in Fig. 1 and Table 1 . Each lin-14 activity is proposed to change from "High" to "Low" during wild-type development (although a change from "On" to "Off" is also consistent with our data; see text). Critical periods, during which the fates of cells are affected by the amount of lin-14 activity, are indicated by the positions of the words "High" and "Low" with respect to the time axis at left and refer to the ts periods for lin-14a and lin-14b as shown in Figs. 4, 5, and 6. Abnormal levels of activity at specified stages and the resulting heterochronic cell fates are shown with open-faced type. Shown here are the minimal number of gene activities required to specify the fates observed in the L1, L2, and L3 stages of the wild type and the mutants. For example, in these mutants only two fates have been observed in the L1 stage, S1 (in the wild type) and S2 (in lin-14( $\left.a^{-}\right)$mutants). Since loss of lin-14b has no effect on L1 fates, it appears that lin-14b is irrelevant to $\mathrm{L} 1$ cells and alternative levels of lin-14a alone (either high or low) are sufficient to specify which of these fates is expressed; in the wild type, high lin-14a in the early L1 specifies S1 lineage patterns. Since lin-14b is irrelevant at this stage, no level for that function is shown. In contrast, for cells in the L2 stage, three fates can occur, S2 (in the wild type), S1 (in lin-14( $a^{++}$) mutants), and S3 (in lin-14( $\left.b^{-}\right)$mutants). Thus, for cells in the L2 stage, three different combinations of high and low levels of the two lin-14 functions are relevant. In the wild type, S2 lineage patterns are caused by low lin-14a combined with high lin-14b. For L 3 cells, a high or low level of lin-14b is sufficient to choose between the two fates, S3 (in the wild type) or S2 (in lin-14( $b^{++}$) mutants), so only lin-14b is shown in the late L2. For simplicity, the sequence $(\mathrm{S} 3, \mathrm{~S} 4$, alae) is considered as a unit, since these three events seem to be coordinately controlled by lin-14b (see text; Fig. 1 ; Table 1). (b) The second model is presented using the same conventions empolyed in $a$. This model differs from the first in that lin-14a and lin-14b are proposed to reflect the activities at distinct times of a single product with a single biochemical function. According to this model, cells at successive stages distinguish between a low level of lin-14 gene function and two different higher levels of function and express fates appropriately. During wild-type development, a "high" level of $l i n-14$ in the early-L1 specifies S1 lineage patterns, a lower ("medium") level in the late-L1 specifies the subsequent expression of S2 patterns, and a still lower ("low") level in the L2 stage specifies the expression of S3 patterns in the L3. L1 cells express S1 lineage patterns (in the wild type and in class I and class III mutants) in response to a high level of lin-14 and express S2 patterns (in class III, IV or IV mutants) if lin-14 is at a lower level ("low" or "medium"). Cells at the L2 and L3 stages express one of three potential fates, depending upon lin-14 level: S1 if lin-14 is at a high level, S2 if lin-14 is at a medium level, and S3 if lin-14 is at a low level. According to this model, the phenotypes caused by lin-14 mutations result, in certain cases, from changes in the developmental kinetics of lin-14 level (e.g., class I and class III mutants), or, in other cases, from a change in the level of lin-14 at all stages (class II), or from a combination of these two effects (class IV and V). 
that independently decrease in activity during wild-type development (Figs. 8a and 9a). According to this model, the lin-14a product acts during the early-Ll stage to specify whether cells in the L1 express S1 or S2 lineage patterns and lin-14b acts during the late-L1 stage to specify whether cells in the L2 express S2 or S3 lineage patterns. The normal sequence of these events is proposed to be caused by a change in the lin-14a activity from high in the early-Ll to low in the late-Ll and a change in the lin-14b activity from high in the late $\mathrm{L} 1$ to low in the L2. The reiteration of S1 lineage patterns at the L2 stage (class I mutants) or the reiteration of S2 patterns at the L3 stage (class I or class V mutants) is caused by high levels of $l i n-14 a$ or $\operatorname{lin}-14 b$, respectively, at times when those activities are normally low. The precocious expression of S2 patterns in the L1 stage or of S3 patterns in the L2 stage are caused by the loss of the lin-14a or lin-14b activities, respectively. The coordinate loss of both activities (class II mutants) results in the precocious expression of both of these fates. The independent loss of either lin-14a (class IV mutants) or lin-14b (class III mutants) results in the expression of either precocious $\mathrm{S} 2$ only or precocious $\mathrm{S} 3$ only.

An alternative model is that $\operatorname{lin}-14 a$ and $\operatorname{lin}-14 b$ reflect the activities at distinct times during development

a. Model 1: two products, two levels of each.

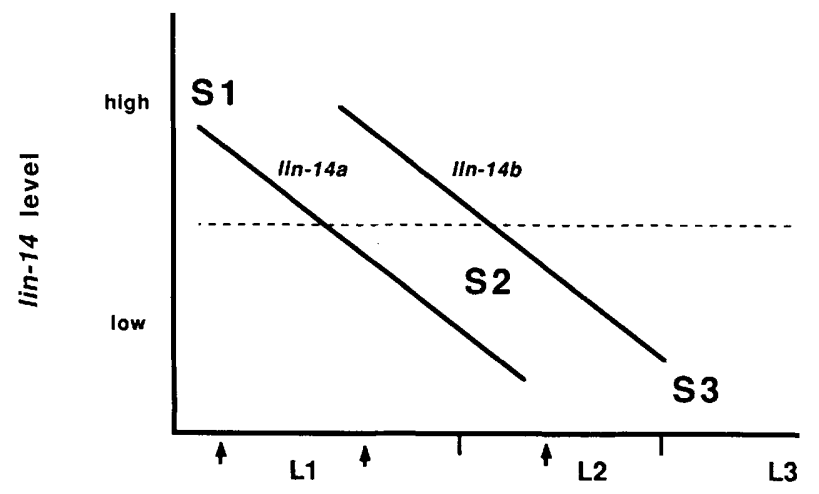

b. Model 2: one product, three levels.

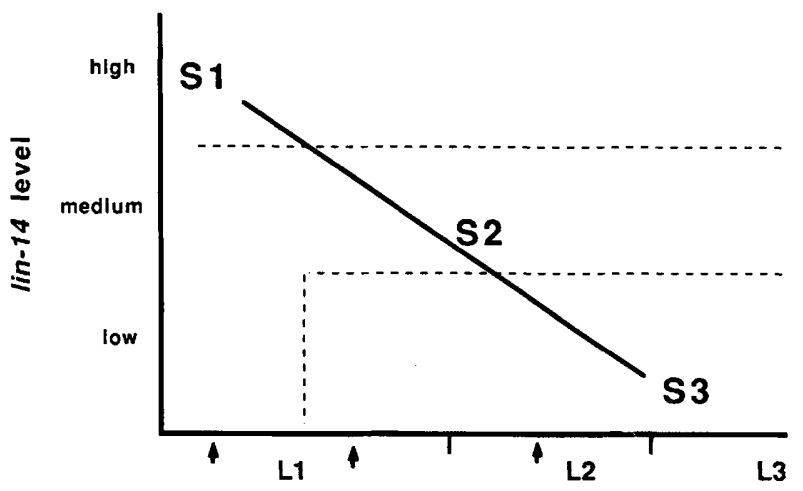

of a single gene product with a single biochemical function. According to this model, cells at successive stages can distinguish among three levels of $\operatorname{lin}-14$ activity (low, medium, high) and express fates accordingly (Figs. $8 \mathrm{~b}$ and $9 \mathrm{~b}$ ). During wild-type development, a high level of lin-14 in the early-L1 specifies S1 lineage patterns, a medium level in the late-L1 stage specifies S2 patterns in the L2 stage, and a low level in the L2 stage specifies the expression of S3 patterns in the L3 stage. Cells in the L1 stage express one of two potential fates, depending on lin-14 activity: S1 lineage patterns (in the wild type and in class I and class III mutants) in response to a high level of lin-14 activity and S2 patterns (in class II, IV, or $\mathrm{V}$ mutants) if lin-14 activity is at a lower level (either low or medium). Cells at the L2 and L3 stages express one of three potential fates: S1 if lin-14 activity is high, S2 if medium, and S3 if low. According to this model, the phenotypes caused by lin-14 mutations result, in certain cases, from changes in the temporal regulation of lin-14 activity (e.g., class I and class III mutants), or, in other cases, from a change in the level of lin-14 at all stages (class II), or from a combination of these two effects (classes IV and V). Class IV and class V alleles were generated by reversion of class I alleles, suggesting that the alterations in temporal regulation proposed for these

Figure 9. A graphic depiction of the two models proposed in Fig. 8 for the stage-specific action of lin-14 during the first three larval stages of wild-type development. Parts $a$ and $b$ of this figure correspond to the first panels of Fig. 7, $a$ and $b$, respectively. For simplicity, the proposed temporal changes in the level of lin-14 are shown to follow linear kinetics. The arrows indicate the approximate times of the critical periods for each cell fate decision and refer to the ts periods shown in Figs. 4, 5, and 6. (a) Model 1 proposes that $\operatorname{lin}-14 a$ and $\operatorname{lin}-14 b$ reflect the activities of functionally distinct lin-14 products that are independently regulated. According to this model, levels of gene function above a threshold (signified by the dotted line), are effectively high, and levels below the threshold are effectively low. For cells in the L1 stage, fates are selected based on the level of lin-14a only: A high level of lin-14a selects S1 lineage patterns instead of S2 patterns. For cells in the L2 stage, a low level of lin-14a combined with a high level of lin-14b selects S2 patterns instead of S1 or S3 patterns. For cells in the L3 stage, a low level of both lin-14a and lin-14b selects S3 patterns instead of S1 or S2 patterns. (b) Model 2 proposes that a single lin-14 gene product exists and that cells distinguish among three functional levels of this product. Levels of gene function above the upper threshold are effectively high, levels below the lower threshold are effectively low, and levels between the two thresholds are effectively medium. For cells in the L1 stage, a high level of lin-14 selects S1 lineage patterns instead of S2 patterns. For cells in the L2 stage, a medium level of lin-14 selects S2 patterns instead of S1 or S3 patterns. For cells in the L3 stage, a low level of lin-14 selects S3 patterns instead of S1 or S2 patterns. The lower threshold is depicted as nonexistent in the L1 stage to account for the observation that there seem to be only two effective levels of $l i n-14$ for cells in the L1 stage; i.e., in mutants any level of lin-14 less than a high level results in S2 patterns instead of S1 patterns. In contrast, in the $\mathrm{L} 2$ and $\mathrm{L} 3$ stages, one of three potential fates can occur, S1, S2, or S3 patterns, depending upon lin-14 level. 
classes of alleles could result from a simple alteration in kinetics caused by a class I mutation combined with a partial reduction in lin-14 level at all stages caused by a weak class II mutation.

The complementation observed in inter-allelic heterozygotes containing a class III and a class IV allele (Table 3 ) is consistent with both models. According to the first model, this complementation reflects two different functional gene products, each supplied by a separate allele. According to the second model, the complementation results from the combined temporal kinetics of two different allelic copies of the same product, each copy of which is specifically reduced in level at a different stage; the net result is a functionally normal level of $l i n-14$ activity throughout development of the heterozygote.

The simplest explanation for the phenotypes of lin-14 mutants and the results of our temperature-shift experiments is that lin-14 levels decrease during wild-type development. Alternatively, the level of the lin-14 product(s) may be constant and instead the level of some interacting component may change.

A molecular analysis of 1 in-14 could both test our proposed model that lin-14 activity is temporally regulated and also distinguish between our alternative explanations for the stage-specific activities we have defined as lin-14a and lin-14b. The functional complexity of the lin-14 locus could reflect separate gene products, separate domains of a multifunctional single product, or different processed forms of the same primary RNA or protein product (consistent with model 1, Figs. 8a and 9a). Alternatively, the stage-specific lin-14 functions could reflect the temporal regulation of a single product (model 2, Figs. 8b and 9b). To address these and other questions concerning the nature of the 1 in -14 product(s), the developmental expression of lin-14, and the molecular relationship between the lin-14a and lin-14b activities, we are now isolating and analyzing molecular clones of the lin-14 locus (G. Ruvkun, V. Ambros, and R. Horvitz, unpubl.).

Similarities between lin-14 and other genes that control developmental patterns

We have proposed that the normal temporal pattern of expression of cell fates in the lateral hypodermal cell lineages is caused by temporal changes in the level of lin-14 gene activity. The principal evidence supporting this hypothesis is the finding that mutations that reduce lin-14 activity at early stages of development lead to transformations in cell fates opposite to those caused by mutations that appear to elevate lin-14 activity at later stages. This result indicates that $\operatorname{lin}-14$ wild-type gene activity is necessary for the acquisition of specific developmental fates for those cells that normally express those fates and is sufficient for the acquisition of those same fates by cells at other times in the same cell lineage.

These general properties of $\operatorname{lin}-14$ are analogous to those of homeotic genes thought to control spatial patterns of cell fates. For example, mutations that elevate activity of the C. elegans gene lin-12 transform the fates of certain cells into the fates of cells located elsewhere, while mutations that eliminate lin-12 cause the reciprocal transformations in cell fates (Greenwald et al. 1983). For the cells affected, lin-12 wild-type activity appears to be both necessary and sufficient for the acquisition of normally spatially specific fates. During wildtype development, different levels of $\operatorname{lin}-12$ activity appear to cause different cells to express different fates. Mutations of the Antennapedia (Antp) locus of Drosophila melanogaster display properties similar to mutations of lin-14 and lin-12. Dominant mutations /which appear to cause inappropriate expression of Antp activity) transform antennae into legs, while recessive mutations (which appear to cause loss of gene activity) result in the opposite transformation of legs to antennae (Struhl 1981; Hazelrigg and Kaufman 1983). The differential expression of Antp activity between the head and the mesothorax is thought to specify the wild-type segmental location of antennae and legs. A positional control of homeotic gene function resulting in the spatial patterning of different cell fates is analogous to a temporal control of lin-14 function leading to the temporal patterning of cell fates.

The genetic properties and phenotypic effects of mutations in loci such as lin-14, lin-12, and Antp may provide criteria for the identification of genes that play important roles in controlling developmental patterns: Mutations that elevate gene activity cause transformations in developmental fates opposite to those that reduce gene activity. Such genes may specify which developmental programs are expressed by cells that share multiple potential fates. These genes could control patterns of development by being expressed differentially, for example, in space (lin-12 and Antp) or in time (lin-14).

\section{Materials and methods \\ General methods and strains}

General methods for the culturing and handling of $C$. elegans have been described by Brenner (1974). Methods used for Nomarski differential interference contrast microscopy of living animals and for photography have been described by Sulston and Horvitz (1977) and Sternberg and Horvitz (1981). Cells are named based on their descent from postembryonic blast cells as described by Sulston and Horvitz (1977).

The wild-type parent of all strains used in this work is Caenorhabditis elegans var. Bristol strain N2. The genetic markers listed below are from the Cambridge collection (Brenner 1974) unless otherwise noted. lin-14 mutations were isolated as described by Ambros and Horvitz (1984). This paper conforms to standard C. elegans genetic nomenclature (Horvitz el al. 1979). LGIII, dpy-17 (e164), tra-1 (e1099) (Hodgkin and Brenner 1977); LGV, him-5 (e1467) (Hodgkin et al. 1979); LGX, dpy-7 (e1324), dpy-6 (e14), unc-9 (e101). The reciprocal translocation $s z T 1$ $(X ; I)$ is a balancer for LGX that causes recessive lethality in hermaphrodites and carries a wild-type allele of lin-14 (Fodor and Deak 1985). The deficiency $n D f 19$ is a deficiency that deletes the lin-14 locus (Ambros and Horvitz 1984).

\section{Complementation tests}

For the complementation tests reported in Table 3, heteroallelic strains were constructed using tra-1 XX males (hemi- 
zygous, $\mathrm{XO}$, males carrying most lin-14 alleles are defective in mating). tra-1; lin-14(recessive)/ + males were mated with hermaphrodites of the following genotypes: $d p y-17 ; s z T 1(X ; I) /$ $n D f 19, d p y-17 ; \quad s z T 1(X ; I) /$ lin-14(n536 n540), and dpy-17; lin-14(n360). Cross progeny (non dumpy) hermaphrodites were screened using Nomarski differential interference contrast optics for heterochronic defects (see below). A number of cross progeny of each phenotypic class were progeny tested to verify their genotypes.

Animals were examined at defined developmental stages using Nomarski optics to identify the fates of individual cells as described below. $\mathrm{Ll}$ animals were examined for the precocious expression of normally L2-specific cell division patterns (S2 lineage segments) in the lateral hypodermis. Animals at L3 lethargus were examined for precocious adult alae formation. Generally, for each heteroallelic combination, 10 or more animals were examined (usually one side of each).

\section{Gene dosage experiments}

For the gene dosage experiments described in Table 2, strains were constructed as follows: $n D f 19 /+$ animals were obtained from among the self-progeny of $n D f 19 / s z T 1$ hermaphrodites. $n 179 / n D f 19$ animals were obtained as hermaphrodite crossprogeny (non dumpy) of him-5; lin-14(n179ts) males (grown at $15^{\circ} \mathrm{Cl}$ and $d p y-17$; $s z T 1 / n D f 19$ hermaphrodites. Hermaphrodites that did not display any precocious defects were progeny tested at $25^{\circ} \mathrm{C}$ and the results were consistent with the genotype $d p y-17 /+$; him-5/+; szT1/lin-14(n179ts). Similar procedures were used to obtain $n 179 / n 536 n 540$ animals.

The lin-14(n355 n679) homozygous animals screened in experiments 1 and 2 of Table 4 were siblings of the heterozygous animals screened in the same experiments. In experiment 3 , lin-14(n355 n679) animals were derived from lin-14(n355 n679) homozygotes. lin-14(n355 n679)/ + animals were obtained by different methods in experiments 1,2 , and 3. Experiment 1 : Wild-type males were mated at $15^{\circ} \mathrm{C}$ with lin-14 (n355 n679) hermaphrodites, and their hermaphrodite progeny were screened at the L4 molt to determine the frequency of adult alae formation by seam cells; these animals then were progeny tested to confirm their genotypes. Experiment 2: Non dumpy self-progeny from $d p y-6 /$ lin-14(n355 n679) hermaphrodites were similarly screened at the L4 molt and progeny tested. Experiment 3: Non dumpy self-progeny from dpy-6 lin-14/n355 n679)/dpy-7 unc-9 hermaphrodites were screened at the L4 molt and progeny tested. lin-14(n355 n679)/lin-14(n536 n540) animals and lin-14(n355 n679)/lin-14(n360) animals were identified by screening (for the frequency of adult alae formation at the $\mathrm{L} 4 \mathrm{molt}$ ) random self-progeny from hermaphrodites of the same respective genotypes and progeny testing each screened animal to confirm its genotype. lin-14(n536)/lin-14(n536) animals and lin-14(n536)/ + animals were obtained by screening (for alae formation at the L4 molt) random self-progeny of lin-14(n536)/szT1 hermaphrodites. lin-14(n536)/lin-14(n536 n540) animals were obtained by similarly screening random self-progeny of lin-14(n536)/lin-14(n536 n540) hermaphrodites. For both strains, the genotype of each screened animal was determined by progeny testing. To ensure that a random sample of animals of each genotype was screened, parents were transferred daily and all progeny produced on 1 day were screened. To maximize temperature uniformity, cultures containing animals to be screened in each experiment were kept in close proximity in the same incubator and handled in parallel.

Living hermaphrodites were observed using Nomarski optics (Sulston and Horvitz 1977) at defined stages of development. All seam cells positioned between the posterior bulb of the pharynx and the anus were observed, and their developmental fates were determined as described below.

\section{Temperature-shift experiments}

Two temperature-sensitive alleles, lin-14(n179ts) and lin-14(n355n679ts), were used in the temperature-shift experiments shown in Figures 4, 5, and 6. lin-14(n179ts)/lin-14(n360) animals were generated by mating him-5; lin-14 (n179ts) males with dpy-17; lin-14(n360) hermaphrodites and selecting non dumpy cross-progeny. Separate crosses were performed at $15^{\circ} \mathrm{C}$ and $25^{\circ} \mathrm{C}$. Individual animals were picked from cultures grown at one temperature, examined by Nomarski optics $\left(20^{\circ} \mathrm{C}\right)$ to determine developmental stage according to the criteria described below, immediately placed in Petri dishes on agar at either the same temperature or the alternative temperature, and then were maintained at that temperature for the remainder of their development. Some animals ("pulse-shifted" animals) were transferred back to the original temperature and then maintained at that temperature for the remainder of their development. The total time involved in staging and shifting each animal was less than $10 \mathrm{~min}$.

Animals were examined at $20^{\circ} \mathrm{C}$ using Nomarski optics either before the $\mathrm{L} 1 \mathrm{molt}$ to infer the patterns of cell divisions and progeny cell types generated during the Ll stage and/or shortly after the Ll molt to infer the patterns of cell divisions that occurred at the $\mathrm{Ll}$ molt as described below. The presence of alae on the cuticles of L4 or L5 animals was scored using Nomarski optics at $1000 \times$ or incident illumination at $50 \times$ in a dissecting microscope. A quantitative assessment of the strength of mutant phenotypes for each animal was derived by determining the proportion of seam cells in that animal that expressed a heterochronic fate at a specified stage.

\section{Determining developmental stage}

We have used a variety of developmental events that are not affected in lin-14 mutants as markers for determining developmental stage. Animals were examined using Nomarski microscopy for the occurrence of specific cell divisions and for the presence of specific cells and/or morphological structures. The following developmental events were used to delineate temporal segments of the $\mathrm{L} 1$ and $\mathrm{L} 2$ stages (times that these events occur are given in hours after hatching at $20^{\circ} \mathrm{C}$, as described by Sulston and Horvitz 1977): $3 \mathrm{hr}$, division of Q; $4.5 \mathrm{hr}$, division of V5; $6 \mathrm{hr}$, division of Q.a and Q.p; $6.5 \mathrm{hr}$, division of $\mathrm{M} ; 7.5 \mathrm{hr}$, migration of $\mathrm{Pl} ; 8 \mathrm{hr}$, migration of $\mathrm{P} 6$ and divisions of M.d and M.v; 9 hr, migration of P12; $10.5 \mathrm{hr}$, division of P6.p and third round of $\mathrm{M}$ lineage divisions; $12 \mathrm{hr}$, third round of divisions in the $\mathrm{P} 6$ lineage, $13 \mathrm{hr}$, programmed cell deaths in the $\mathrm{P} 9$ and $\mathrm{Pl} 0$ lineages; $14 \mathrm{hr}$, beginning of $\mathrm{L} 1$ lethargus; $15.5 \mathrm{hr}$, V5.p division; $16 \mathrm{hr}$, Ll ecdysis; $16 \mathrm{hr}$, V6.p division; $17.5 \mathrm{hr}$, divisions of $\mathrm{V}(1-4)$.pa and V(1-4).pp; $19 \mathrm{hr}$, V5.pap division; $22 \mathrm{hr}$, V5.paapp programmed cell death; $23 \mathrm{hr}$, beginning of L2 lethargus; $25 \mathrm{hr}$, $\mathrm{L} 2$ ecdysis. The developmental stage of $\mathrm{L} 1$ and $\mathrm{L} 2$ animals was assumed to be at the time of one of these events (for animals in which an event was observed to be occurring) or at a time midpoint between two events. Third- and fourth-stage animals were distinguished by the extent of gonad development, including the number of somatic and germ line nuclei and extent of morphogenesis (Kimble and Hirsh 1979). The presence of fertilized oocytes in the gonad distinguishes young adult (fifth stage) hermaphrodites from L4 larvae. These staging criteria were confirmed as reliable after following continually or intermittently the development of several mutant individuals. The overall rate of development of most lin-14 mutants is comparable to that of the wild type, although the development of the five severe class II mutants (e.g., n536 n540) is significantly slower than that of the wild type (Ambros and Horvitz 1984). 


\section{Scoring seam cell fates}

Generally an individual seam cell in a lin-14 mutant expresses either its normal fate or the fate of a cell earlier or later in the same lineage. The developmental fate of a blast cell is defined by the cell lineage pattern that it generates. Characteristics of a lineage pattern are the lineal relationships among a set of progeny cells, the polarity of each cell division, and the specific cell types produced by each division. Cell types are scored by nuclear morphology as seen using Nomarski optics (Sulston and Horvitz 1977). The stage-specific cell lineage patterns affected by lin-14 mutations are known as S1, S2, S3, and S4, referring to the larval stages in which they normally occur (L1, L2, L3, and L4 stages, respectively) (Ambros and Horvitz 1984). Occasionally, we observed cell fates in mutants different from any wild-type fate. In the lateral hypodermis, such abnormal fates were rare (fewer than $5 \%$ of the cells examined) and are not considered in this manuscript.

Seam cells normally cease cell division and form adult-specific cuticular structures known as lateral alae toward the end of the L4 stage (Sulston and Horvitz 1977; Singh and Sulston 1978). Nomarski optics can be used to visualize alae on living animals; alae lie superficial to the seam cells that form them and are normally continuous along the length of each side of the animal (see Fig. 3d). Most heterochronic mutants are of variable expressivity in that some seam cells on one side of an animal may lie beneath lateral alae, while the rest may lie beneath gaps in the alae. We have assumed that those seam cells beneath gaps do not participate in alae formation. Our observations suggest that seam cells not forming adult alae always undergo cell division in these mutants. The seam cells beneath segments of alae appear to have ceased cell division (see Fig. 2). The formation of adult alae by individual seam cell nuclei are scored by selecting animals during lethargus, when newly formed alae are distinct, and observing these animals using Nomarski optics. For each animal, the total number of seam cells between the posterior bulb of the pharynx and the anus on one side was counted, and the number of those cells lying under newly formed alae was determined. (Seam cells that were undergoing cell division or that had recently completed cell division at the time of observation were counted as one seam cell.) Several animals of each genotype were examined.

The time of expression of stage-specific cell division patterns was assayed by the continual observation of cell lineages or by anatomical screens at defined stages of development. The ease and reliability of anatomical screening is based on three properties of C. elegans development: (1) The developmental stage of an animal can be readily and precisely determined (see above). (2) Newly formed lateral hypodermal nuclei can be identified within an hour or two after their births by their characteristic morphology and smaller size relative to older cells of the same type. (3) Because newly formed sister cells generated by S2 lineage patterns of the $\mathrm{V} 1-\mathrm{V} 4$ and $\mathrm{V} 6$ lineages remain near each other in a characteristic spatial arrangement, we can distinguish the products of an S2 cell division pattern from those of an S1 pattern, and we can distinguish the products of an S2 pattern from those of an S3 pattern. We have utilized anatomical screens to score the expression of $\mathrm{S} 1$ versus $\mathrm{S} 2$ patterns in the L1, L2, and L3 stages, the expression of S2 versus S3 patterns in the L2 and L3 stages, and whether seam cells at the L3, L4, and L5 molts form adult alae or undergo cell division.

\section{Acknowledgments}

We thank Edwin Ferguson, Meredith Kusch, and Nancy Tsung for providing lin-14 mutants. We are grateful to Robert Edgar,
Edwin Ferguson, Michael Finney, William Fixsen, Iva Greenwald, Stuart Kim, and Paul Sternberg for valuable advice and discussions. We thank Rosalind Lee, Doug Melton, Dan Stinchcomb, Gary Struhl, and members of our laboratories for comments on the manuscript. This work was supported by Public Health Service research grants GM24663 (H.R.H.), GM24943 (H.R.H.), and GM34028 (V.A.), Public Health Service Research Career Development Award HD00369 (H.R.H.), National Institutes of Health postdoctoral fellowship F32 GM08642 (V.A.), and American Cancer Society fellowship NYPF-1726 (V.A.).

\section{References}

Ambros, V. and H.R. Horvitz. 1984. Heterochronic mutants of the nematode Caenorhabditis elegans. Science 266: 409416.

Brenner, S. 1974. The genetics of C. elegans. Genetics 77: 7194.

Chalfie, M., H.R. Horvitz, and J.E. Sulston. 1981. Mutations that lead to reiterations in the cell lineages of Caenorhabditis elegans. Cell 24: 59-69.

Cline, T.W. 1984. Autoregulatory functioning of a Drosophila gene product that establishes and maintains the sexually determined state. Genetics 107: 231-271.

Fodor, A. and P. Deak. 1985. The isolation and analysis of a $C$. elegans translocation (szT1) strain bearing an X-chromosome balancer. I. Genet. 64: 143.

Greenwald, I.S., P.W. Sternberg, and H.R. Horvitz. 1983. The lin-12 locus specifies cell fates in Caenorhabditis elegans. Cell 34: 435-444.

Hazelrigg, T.I. and T.C. Kaufman. 1983. Revertants of dominant mutations associated with the Antennapedia gene complex of Drosophila melanogaster: Cytology and genetics. Genetics 105: 581-600.

Hodgkin, J. and S. Brenner. 1977. Mutations causing transformations of the sexual phenotype of the nematode Caenorhabditis elegans. Genetics 86: 275-287.

Hodgkin, J., H.R. Horvitz, and S. Brenner. 1979. Nondisjunction mutants of the nematode Caenorhabditis elegans. Genetics 91: 67-94.

Horvitz, H.R., S. Brenner, J. Hodgkin, and R. Herman. 1979. A uniform genetic nomenclature for the nematode Caenorhabditis elegans. Mol. Gen. Genet. 175: 129-133.

Kimble, J. and D. Hirsh. 1979. Postembryonic cell lineages of the hermaphrodite and male gonads in Caenorhabditis elegans. Dev. Biol. 70: 396-417.

Morata, G. and P.A. Lawrence. 1977. Homoeotic genes, compartments, and cell determination in Drosophila. Nature 265: $211-216$.

Ouweneel, W.J. 1976. Developmental genetics of homoeosis. Adv. Genet. 18: 179-248.

Singh, R. and J.E. Sulston. 1978. Some observations on moulting in Caenorhabditis elegans. Nematologica 24: 6371.

Sternberg, P.W. and H.R. Horvitz. 1981. Gonadal cell lineages of the nematode Panagrellus redivivus and implications for evolution by modification of cell lineage. Dev. Biol. 88: $147-166$.

1984. The genetic control of cell lineage during nematode development. Annu. Rev. Genet. 18: 489-524.

Struhl, G. 1981. A homoeotic mutation transforming leg to antenna in Drosophila. Nature 292: 635-638.

Sulston, J. and H.R. Horvitz. 1977. Post-embryonic cell lineages of the nematode Caenorhabditis elegans. Dev. Biol. 56: $110-156$. 


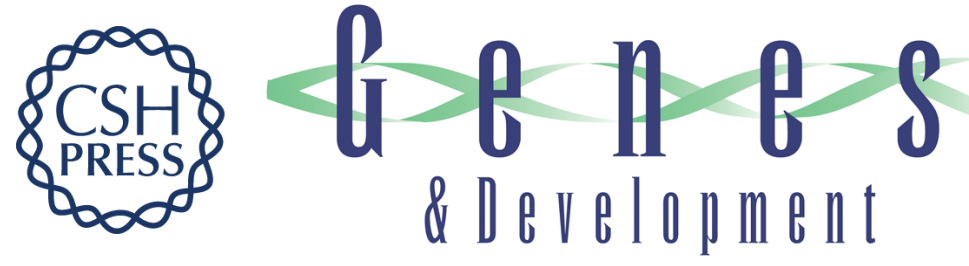

\section{The lin-14 locus of Caenorhabditis elegans controls the time of expression of specific postembryonic developmental events.}

V Ambros and H R Horvitz

Genes Dev. 1987, 1:

Access the most recent version at doi:10.1101/gad.1.4.398

References This article cites 18 articles, 5 of which can be accessed free at: http://genesdev.cshlp.org/content/1/4/398.full.html\#ref-list-1

License

Email Alerting Service right corner of the article or click here.

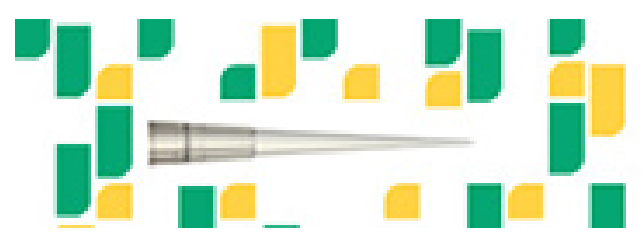

